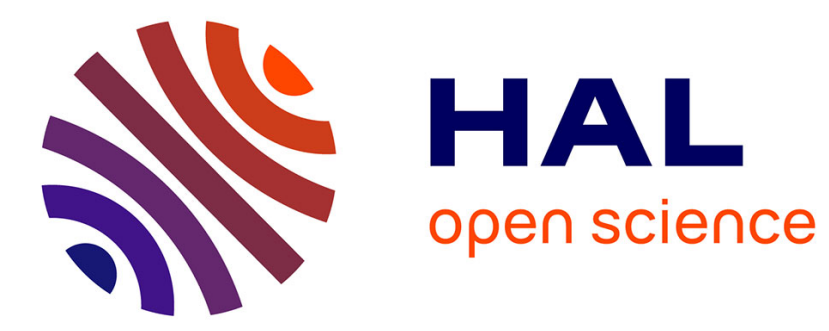

\title{
Studying the Locator Polynomials of Minimum Weight Codewords of BCH Codes
}

\author{
Daniel Augot, Pascale Charpin, Nicolas Sendrier
}

\section{To cite this version:}

Daniel Augot, Pascale Charpin, Nicolas Sendrier. Studying the Locator Polynomials of Minimum Weight Codewords of BCH Codes. IEEE Transactions on Information Theory, 1992, 38 (3), pp.960973. 10.1109/18.135638. hal-00723733

\section{HAL Id: hal-00723733 https://hal.inria.fr/hal-00723733}

Submitted on 13 Aug 2012

HAL is a multi-disciplinary open access archive for the deposit and dissemination of scientific research documents, whether they are published or not. The documents may come from teaching and research institutions in France or abroad, or from public or private research centers.
L'archive ouverte pluridisciplinaire HAL, est destinée au dépôt et à la diffusion de documents scientifiques de niveau recherche, publiés ou non, émanant des établissements d'enseignement et de recherche français ou étrangers, des laboratoires publics ou privés. 


\title{
Studying the Locator Polynomials of Minimum Weight Codewords of $\mathrm{BCH}$ Codes
}

\author{
Daniel Augot, Pascale Charpin, and Nicolas Sendrier
}

\begin{abstract}
Only primitive binary cyclic codes of length $n=$ $2^{m}-1$ are considered. A BCH-code with designed distance $\delta$ is denoted $B(n, \delta)$. A BCH-code is always a narrow-sense $\mathrm{BCH}$ code. A codeword is identified with its locator polynomial, whose coefficients are the symmetric functions of the locators. The definition of the code by its zeros-set involves some properties for the power sums of the locators. Moreover, the symmetric functions and the power sums of the locators are related to Newton's identities. First presented is an algebraic point of view in order to prove or disprove the existence of words of a given weight in a code. The main tool is symbolic computation software to explore Newton's identities. The principal result is the true minimum distance of some BCH-codes of length 255 and 511, which were not known. In a second part, the minimum weight codewords of the codes $B\left(n, 2^{h}-1\right)$ are studied. It is proven that the set of the minimum weight codewords of the BCH-code $B\left(n, 2^{m-2}-1\right)$ equals the set of the minimum weight codewords of the punctured Reed-Muller code of length $\boldsymbol{n}$ and order 2 , for any $m$. Several corollaries of this result are given.
\end{abstract}

Index Terms-Cyclic code, BCH-code, Reed-Muller code, locator polynomial, Newton's identities.

\section{INTRODUCTION}

$\mathbf{I}_{\mathrm{ar}:}^{\mathrm{N}}$ $\mathrm{N}$ this paper, we treat primitive binary cyclic codes. We are going to introduce a method for finding the true minimum distance of these codes.

We will first recall the usual definitions in Section II, as introduced in [11]. We can have an algebraic description of the codewords in a cyclic code, by studying their locator polynomials. This investigation of the locator polynomial of a codeword is achieved via the Newton's identities.

In Section III, we will show how to use the Newton's identities. In fact, we explore the identities in a progressive manner, using symbolic computation software. We have two options: either trying to establish a contradiction, or trying to find an effective solution of the Newton's identities. This method enables us to complete the table of the minimum distance of the $\mathrm{BCH}$ codes of length 255 , and to extend our knowledge of $\mathrm{BCH}$ codes of length 511 . Some of the longer proofs are given in Appendixes A, B, and C.

In Section IV, we give a description of the set of the minimum weight codewords of the $\mathrm{BCH}$ codes of length

Manuscript received December 12, 1990; revised September 12, 1991 This work was presented in part at the IEEE International Symposium on Information Theory, Budapest, Hungary, June 23-28, 1991.

D. Augot is with the Univesité Paris 6, Laboratoire d'Informatique Théorique et Programmation (LITP), 2 pl. Jussieu, 75251 Paris Cedex 05, France.

P. Charpin and N. Sendrier are with the Institut National de Recherche en Informatique et Automatique (INRIA), Domaine de Voluceau, Rocquencourt, BP105, 78153 Le Chesnay Cedex, France.

IEEE Log Number 9105773 $2^{m}-1$ and designed distance $2^{m-2}-1$ (Theorem 6). We prove that the locator polynomials of such codewords are, in fact, linearized polynomials. We obtain this result by studying Newton's identities associated to the minimum weight codewords of the $\mathrm{BCH}$-codes of designed distance $2^{h}-1$, $h \in[2, m-1]$. Certain properties yield a complete characterization when $h=m-2$. When $h \neq m-2$, our proof involves an algorithm for constructing cyclic codes whose minimum weight codewords have linearized locator polynomials.

\section{Presentation and Notations}

Throughout this section we recall the usual conventions and notation as used in [11].

\section{A. The BCH Codes and Their Minimum Distance}

Let $n=2^{m}-1$. We denote by GF ( $q$ ) the Galois field of order $q$, where $q=2^{m}$ and denote by $\alpha$ a primitive $n$th root of unity in GF $(q)$. Any cyclic code $C$ of length $n$ can be defined by its generator polynomial whose roots are called the zeros of the code $C$. Thus, we say that the defining set of $C$ is the set

$$
I(C)=\left\{i \in[0 \cdots n-1] \mid \alpha^{i} \text { is a zero of } C\right\} .
$$

We denote by $\operatorname{cl}(s)$ the cyclotomic class of $s$ modulo $n$ :

$$
c l(s)=\left\{s, 2 s, 2^{2} s, \cdots, 2^{m-1} s \bmod n\right\} .
$$

If $\alpha^{i}$ is a zero of $C$ then $\alpha^{2 i}$ is also a zero of $C$, so $I(C)$ is a union of cyclotomic classes $\mathrm{cl}(s)$.

Thus, we can define the primitive narrow-sense $\mathrm{BCH}$ code of length $n$ of designed distance $\delta$, denoted by $B(n, \delta)$, as the cyclic code of length $n$ whose defining set is the union of the cyclotomic classes $c l(1), \operatorname{cl}(2), \cdots, c l(\delta-1)$. This "designed distance" terminology is used because of the wellknown $\mathrm{BCH}$-bound theorem.

Theorem 1: If the defining set of the cyclic code $C$ contains a set of $\delta-1$ consecutive integers $(0$ is treated consecutive to $n-1$ ), then the minimum distance of $C$ is at least $\delta$.

So the code $B(n, \delta)$ has minimum distance at least $\delta$. We are not satisfied by such a result. Generally the designed distance is equal to the minimum distance, but we have no systematic way of finding the true minimum distance. Of course there exist many other bounds for cyclic codes (J. H. van Lint and R. M. Wilson give an in-depth treatment of the subject in [13]), but these are not necessarily tight 
bounds. It is a difficult problem to find the true minimum distance of long $\mathrm{BCH}$ codes.

The problem one encounters when looking for the true minimum distance is to work with the structure of the finite field GF $(q)$ itself. This structure deeply influences the properties of cyclic codes, and bounds obtained from the properties of the defining set of a cyclic code do not reflect this underlying algebraic structure of GF $(q)$.

\section{B. Mattson-Solomon Polynomial and Locator Polynomial}

Definition 1: The Mattson-Solomon polynomial of the word $\boldsymbol{x}=\left(x_{0}, x_{1}, \cdots, x_{n-1}\right)$ is the polynomial $A \in$ $\mathrm{GF}(q)[Z]$ :

$$
A(z)=\sum_{i=1}^{i=n} A_{i} z^{n-i}
$$

where

$$
A_{i}=x\left(\alpha^{i}\right)=\sum_{j=0}^{j=n-1} x_{j} \alpha^{i j}
$$

Remark:

- $A_{2 i \bmod n}=A_{i}^{2}$

- $A_{i+n}=A_{i}$

So there is only one significant $A_{i}$ for every cyclotomic class.

Definition 2: The locator polynomial $\sigma(Z)$ of a word $x$ is

$$
\sigma(Z)=\prod_{i=1}^{i=w}\left(1-X_{i} Z\right),
$$

where the $X_{i}$ are the elements of GF ( $q$ ) which are not zeros of the Mattson-Solomon polynomial of $x$. They are called the locators of $x$.

Definition 3: The elementary symmetric functions of the locators $X_{1}, X_{2}, \cdots, X_{w}$ are the $\sigma_{i}$ :

$$
\begin{gathered}
\sigma_{i}=(-1)^{i} \sum_{1 \leq k_{1}<k_{2} \cdots<k_{i} \leq w} X_{k_{1}} X_{k_{2}} \cdots X_{k_{i}} \\
\sigma_{0}=1 .
\end{gathered}
$$

We have

$$
\sigma(Z)=\sum_{i=0}^{i=w} \sigma_{i} Z^{i}
$$

The inversion formula of (3) (cf. [11] p. 240) implies that $A\left(\alpha^{j}\right)=x_{j}$. Hence, the zeros of the locator polynomial are the inverses of the locations of the nonzero coordinates of $\boldsymbol{x}$.

In the case of binary codes, locators become very interesting, since binary words can be identified by their locators, and so by their locator polynomial.

We have the following property.

Proposition 1: Let $\boldsymbol{x}$ be a word of length $n$ of weight $\boldsymbol{w}$, with locators $X_{1}, X_{2}, \cdots, X_{w}$. Then $x$ is in the cyclic code with defining set $\left\{\alpha^{i_{1}}, \alpha^{i_{2}}, \cdots, \alpha^{\mathrm{i}_{l}}\right\}$, if and only if the following power sum symmetric functions of its locators are zero:

$$
A_{i_{1}}=A_{i_{2}}=\cdots=A_{i_{1}}=0 \text {. }
$$

Recall that the $k$ th power sum symmetric function of $X_{1}, \cdots, X_{w}$ is

$$
A_{k}=\sum_{i=1}^{i=w} X_{i}^{k}
$$

and is the $k$ th coefficient of the Mattson-Solomon polynomial of $\boldsymbol{x}$.

The following relations known as Newton's identities allow us to study the elementary symmetric functions, knowing the power sum symmetric functions.

Proposition 2: Let $X_{1}, X_{2}, \cdots, X_{w}$ be indeterminates over a field $K, \sigma_{i}$ the elementary symmetric functions of the $X_{i}$ and $A_{i}$ the power sum symmetric functions of the $X_{i}$. Then, we have the following relations:

$$
\begin{aligned}
& r \leq w, \quad I_{r}: A_{r}+\sum_{i=1}^{i=r-1} A_{r-i} \sigma_{i}+r \sigma_{r}=0, \\
& r>w, \quad I_{r}: A_{r}+\sum_{i=1}^{i=w} A_{r-i} \sigma_{i}=0 .
\end{aligned}
$$

\section{The Locator Polynomial and BCH Codes}

From the Newton's identities, we have the following result ([11, ch. 9, Lemma 4 p. 260]).

Lemma 1: Let

$$
\sigma(Z)=\sum_{i=0}^{i=w} \sigma_{i} Z^{i}
$$

be a polynomial over GF $\left(2^{m}\right)$. Then $\sigma(Z)$ is the locator polynomial of a codeword $\boldsymbol{x}$ of $B(n, \delta)$, if and only if

a) $\sigma(Z)$ divides $Z^{n}-1$

b) $i \in[1, \delta-1], i$ odd $\Rightarrow \sigma_{i}=0$.

So we can try to find the true minimum distance of a code $B(n, \delta)$ by finding locator polynomials which satisfy conditions a) and b) of Lemma 1.

D. The Codes $B\left(n, 2^{k}-1\right)$ and Linearized Polynomials

Definition 4: Let $l(Z)$ be a polynomial over GF $\left(2^{\mathrm{m}}\right)$. Then $\mathrm{l}(\mathrm{Z})$ is a linearized polynomial, if and only if

$$
l(Z)=\sum_{i=0}^{i=l} a_{i} Z^{2^{i}}
$$

The interesting point about linearized polynomial is the following proposition ([11, ch. 4 p. 119]).

Proposition 3: $l(Z) \in \mathrm{GF}\left(2^{m}\right)[Z]$ is a linearized polynomial if and only if its zeros (eventually in an extension of GF $\left(2^{m}\right)$ ) form a vector space over GF (2).

Now we can prove as follows that the codes $B(n, \delta)$, $\delta=2^{k}-1$ have minimum distance $\delta$.

a) Let $H$ be a $k$-dimensional subspace of GF $\left(2^{m}\right)$ over GF (2).

b) Then the polynomial: $l(Z)=\Pi_{z \in H}(Z-z)$ is a linearized polynomial.

c) It is easy to check that the polynomial $\sigma(Z)=\Pi_{y \in H}$ $y \neq 0(1-y Z)$ satisfies conditions $a)$ and b) of Lemma 1 .

Definition 5: The punctured Reed-Muller code of length $n$ and order $k$, denoted by $\mathscr{R}(k, m)^{*}$ (cf. [11, p. 383]), is 
the cyclic code of length $2^{m}-1$ with the following defining set:

$$
I\left(\mathscr{R}(k, m)^{*}\right)=\left\{i \in[1 \cdots n-1] \mid w_{2}(i)<m-k\right\},
$$

where $w_{2}(i)$ is the weight of the binary representation of $i$.

It is well known that the locators of any minimum weight codeword of the punctured Reed-Muller code of length $2^{m}-1$ and order $m-k$, together with zero, form a $k$ dimensional GF (2)-subspace. So their locator polynomials have the following form:

$$
\sigma(Z)=\sum_{i=0}^{i=k} \sigma_{2^{k}-2^{i}} Z^{2^{k}-2^{i}} .
$$

In Section IV, we will use such a characterization of the minimum weight codewords of codes $\mathscr{R}(k, m)^{*}$.

\section{The Minimum Distance of Some BCH Codes}

Let $C$ be any cyclic code of length $n . \operatorname{GF}\left(2^{m}\right)$ is the smallest field containing the $n$th roots of unity.

We consider Newton's identities (cf. (9)) written in term of $w$ locators $X_{1}, X_{2}, \cdots, X_{w}$. Note that for a cyclic code $C$, $A_{i}$ is replaced by 0 , for all $i$ in the defining set of $C$.

We call this set of equations, Newton's identities for the code $C$ and for the weight $w$.

We call a set of $A_{i}$ 's and $\sigma_{i}$ 's that verify these identities, such that the polynomial $\sigma(z)=\sum_{i=0}^{w} \sigma_{i} z^{i}$ is square-free and splits in GF $\left(2^{m}\right)$, a solution of this system.

Thus, the existence of solutions to Newton's identities for a code $C$ and a weight $w$ is equivalent to the existence of codewords of weight $w$ in $C$.

We, therefore, have two ways of exploring the identities:

- either we prove the absence of a solution, so that there is no codeword of this weight in the code.

- or we find a solution, and this solution gives us a codeword of the given weight.

We use symbolic computation software to carry out this exploration, enabling us to manipulate the equations in their most general form (some of these have hundreds of terms). The method we use in both cases can roughly be described as follows.

1) We write down Newton's identities for a given code and a given weight.

2) We introduce into the equations the simplifications for the particular code in question.

3) We examine the equations one after another, trying either to express an indeterminate in terms of others, or to find a simple equation involving a small number of indeterminates.

Up to now we have not been able to make this exploration in a fully automatic manner; a user interface is necessary to make a proper choice at the critical stages of the search. There are many possible decisions at Step 3), including the decision to discard too large an equation, and it is difficult to make this choice efficiently within a program.

However, whenever possible we have implemented succes- sive refinements to our program, which now does most of these choices automatically.

We will use the following properties of the $A_{i}$ 's.

- $A_{i}^{2}=A_{2 i}$ and $A_{i+n}=A_{i}$, so there is exactly one relevant $A_{i}$ for each cyclotomic class.

- $A_{i}^{2^{m^{\prime}}}=A_{i}$ where $m^{\prime}$ is the cardinal of the cyclotomic class of $i$ (this is a consequence of the previous property).

- If a codeword is shifted, each $A_{i}$ is multiplied by $\alpha^{i}$ where $\alpha$ is the $n$th root of unity chosen for the definition of the code. So, since $A_{w} \neq 0$, if $n$ and $w$ are relatively prime one can suppose that $A_{w}=1$.

\section{A. The Minimum Distance is Known for All Narrow-Sense} Primitive Binary $B C H$ Codes of Length 255

Theorem 2: All the narrow-sense primitive binary $\mathrm{BCH}$ codes of length 255 have their minimum distance equal to their designed distance except

- $B(255,61)$, which has minimum distance 63 ,

- $B(255,59)$, which has minimum distance 61 .

Proof: From [4]-[6], [11], we know that all narrowsense primitive $\mathrm{BCH}$ codes of length 255 reach the $\mathrm{BCH}$ bound except $B(255,61)$ and $B(255,59)$.

For both of these codes we are able to produce words of weight $\delta+2$. Indeed

- $B(255,61) \supset B(255,63)$, and the latter code has minimum weight 63 ,

- for $B(255,59)$, Dornstetter gives in [5] a word of weight 61

Since the minimum distance of primitive $\mathrm{BCH}$ codes is odd, all we have to prove is that there are no codewords of weight 61 (resp. 59) in the code $B(255,61$ ) (resp. $B(255,59))$. These results were obtained from a MAPLE program; traces of the computations are given in Appendixes $\mathrm{A}$ and $\mathrm{B}$.

B. The Minimum Distance is Known for Most Narrow-Sense Primitive Binary BCH Codes of Length 511

We found another code whose minimum distance exceeds the $\mathrm{BCH}$ bound.

Theorem 3: The code $B(511,123)$ has minimum distance $d=127$.

Proof:

a) $B(511,123)$ is included in the punctured Reed-Muller code $\mathscr{R}(4,9)^{*}$ (cf. Definition 5), and so has no codeword of weight 125 , since $125 \equiv 1 \bmod 4$ [11, Corollary 13, p. 447];

b) $B(511,127) \subset B(511,123)$, and $B(511,127)$ reaches the $\mathrm{BCH}$ bound.

From a) and b), we deduce easily that the minimum distance of $B(511,123)$ is 123 or 127 . 

123.

We show in Appendix $\mathrm{C}$ that there is no word of weight

For codes of length 511 we also carried out another kind of search from Newton's identities: finding particular solutions by restricting the field of search. We introduced the following simplifications in the equations: all the $A_{i}$ 's and $\sigma_{i}$ 's are equal to 0 or 1 . From the following lemma, this is exactly the same as looking for the idempotents of given weight.

Definition 6: The support of a word $x \in \operatorname{GF}(q)^{n}$ is the set of its nonzero positions. We denote it by $\operatorname{supp}(\boldsymbol{x})$.

Note that the support of a word of a cyclic code is the set of exponents of its locators.

Lemma 2: Let $C$ be a binary cyclic code of length $n$, and let GF $\left(2^{m}\right)$ be the smallest field containing an $n$th root of unity. Let $\boldsymbol{x}$ be a word of $C$. The following assertions are equivalent:

a) $x$ is an idempotent,

b) the support of $\boldsymbol{x}$ is the union of cyclotomic classes (in GF $\left.\left(2^{m}\right)\right)$,

c) the coefficients of the locator polynomial of $\boldsymbol{x}$ (the $\sigma_{i}$ 's) are in GF (2),

d) the power sum symmetric functions of $\boldsymbol{x}$ (the $A_{i}$ 's) are in GF (2).

Proof:

a) $\Rightarrow$ b) we have $x=x^{2}$. For any $i$,

$$
i \in \operatorname{supp}(x) \Rightarrow 2 i \in \operatorname{supp}\left(x^{2}\right)=\operatorname{supp}(x) \text {. }
$$

So if $i \in \operatorname{supp}(x)$ then $\operatorname{cl}(i) \subset \operatorname{supp}(x)$.

b) $\Rightarrow$ c) The roots of the locator polynomial $\sigma(z)$ are the inverses of the locators, so the set of the exponents of the roots is the union of cyclotomic classes and therefore, $\sigma(z) \in \mathrm{GF}(2)[z]$.

c) $\Rightarrow$ d) If the $\sigma_{i}$ 's are given and are in GF (2), then by induction, using Newton's identities, all the $A_{i}$ 's are in GF (2).

d) $\Rightarrow$ a) Let $A$ be the Mattson-Solomon polynomial of $\boldsymbol{x}$. We have [11, Theorem 22, p. 240]:

$$
\begin{aligned}
\boldsymbol{x}^{2} & =\boldsymbol{x} \text { (as polynomial) } \quad \Rightarrow A^{*} A \\
& =A \text { (component wise product) }
\end{aligned}
$$

Since $A_{j}^{2}=A_{j}$ for all $j$, we have $A^{*} A=A$, and thus, $x^{2}=x$.

This lemma is useful in two ways: it gives a way to find the idempotents from Newton's identities and a way to describe very simply an idempotent by giving its support as a union of cyclotomic classes.

We are able to find idempotents of given weight in some codes. For this search we give values in GF (2) for some of the nonzero $A_{i}$ 's ( 8 of them for instance), and then the set of equations usually becomes easy to solve. It is possible to implement this exploration in a fully automatic manner.

Theorem 4: The code $B(511, \delta)$ contains idempontents of weight $\delta$ or $\delta+1$ for

$$
\delta=19,39,45,53,57,79,83,91,103 .
$$

Proof: We look for codewords with power sum symmetric functions in GF (2). From Lemma 2, these words are idempotents, and they are fully described by the cyclotomic classes partitioning their supports.

We give here, for a designed distance $\delta$, the support of a codeword $x$ of weight $\delta$ or $\delta+1$ :

$$
\delta=19, \quad \omega(x)=19,
$$

$\operatorname{supp}(x)=\operatorname{cl}(0) \cup \operatorname{cl}(23) \cup \operatorname{cl}(91)$,

$$
\delta=39, \omega(x)=39,
$$

$\operatorname{supp}(\boldsymbol{x})=\operatorname{cl}(63) \cup \operatorname{cl}(87) \cup \operatorname{cl}(117)$

$$
\cup \operatorname{cl}(127) \cup \operatorname{cl}(219) \text {, }
$$$$
\delta=45, \quad \omega(x)=45,
$$

$\operatorname{supp}(x)=\operatorname{cl}(17) \cup \operatorname{cl}(37) \cup \operatorname{cl}(57) \cup \operatorname{cl}(93) \cup \operatorname{cl}(103)$,

$$
\delta=53, \omega(x)=54,
$$

$\operatorname{supp}(x)=\operatorname{cl}(17) \cup \operatorname{cl}(31) \cup \operatorname{cl}(41) \cup \operatorname{cl}(45)$

$$
\cup \operatorname{cl}(103) \cup \operatorname{cl}(117),
$$

$\delta=57, \omega(x)=57$,

$\operatorname{supp}(x)=c l(29) \cup \operatorname{cl}(43) \cup \operatorname{cl}(51) \cup \operatorname{cl}(55)$

$$
\cup \operatorname{cl}(61) \cup \operatorname{cl}(63) \cup \operatorname{cl}(219),
$$

$\delta=79, \quad \omega(x)=79$,

$$
\begin{aligned}
\operatorname{supp}(x)= & c l(0) \cup \operatorname{cl}(3) \cup \operatorname{cl}(13) \cup \operatorname{cl}(39) \\
& \cup \operatorname{cl}(41) \cup \operatorname{cl}(61) \cup \operatorname{cl}(73) \\
& \cup \operatorname{cl}(77) \cup \operatorname{cl}(107) \cup \operatorname{cl}(117) \cup \operatorname{cl}(219) \\
\delta= & 83, \quad \omega(x)=84, \\
\operatorname{supp}(x)= & c l(11) \cup \operatorname{cl}(15) \cup \operatorname{cl}(23) \cup \operatorname{cl}(43) \\
& \cup \operatorname{cl}(53) \cup \operatorname{cl}(79) \cup \operatorname{cl}(123) \\
& \cup \operatorname{cl}(183) \cup \operatorname{cl}(191) \cup \operatorname{cl}(219), \\
\delta= & 91, \quad \omega(x)=91, \\
\operatorname{supp}(x)= & c l(0) \cup \operatorname{cl}(7) \cup \operatorname{cl}(13) \cup \operatorname{cl}(25) \\
& \cup \operatorname{cl}(37) \cup \operatorname{cl}(41) \cup \operatorname{cl}(59) \cup \operatorname{cl}(61) \\
& \cup \operatorname{cl}(117) \cup \operatorname{cl}(175) \cup \operatorname{cl}(239), \\
\delta= & 103, \quad \omega(x)=103, \\
\operatorname{supp}(x)= & c l(0) \cup \operatorname{cl}(7) \cup \operatorname{cl}(13) \cup \operatorname{cl}(19) \\
& \cup \operatorname{cl}(27) \cup \operatorname{cl}(31) \cup \operatorname{cl}(87) \\
& \cup \operatorname{cl}(91) \cup \operatorname{cl}(95) \cup \operatorname{cl}(191) \\
& \cup \operatorname{cl}(219) \cup \operatorname{cl}(223) \cup \operatorname{cl}(255) .
\end{aligned}
$$

Since the true minimum distance $d$ is odd, showing that a word has weight $\delta+1$ is sufficient to prove that $d=\delta$.

\section{Remarks:}

- The weight of an idempotent cannot be an arbitrary integer, this integer has to be a sum of the cardinalities of some cyclotomic classes. For instance in GF (512) we have one class with one element, 2 classes with 3 , and 
57 with 9. So the weight of an idempotent is a multiple of 9 plus $0,1,3,4,6$ or 7 (each class can be used once). For instance, 29 cannot be the weight of an idempotent.

- We did not find an idempotent for every possible weight, but this is not surprising; the surprise is that we did find some. Since the set of idempotents and the set of minimum weight words are (very) small, their intersection should be empty most of the time.

Some other minimum distance are known for length 511. Table I gives a list of them as well as the way they were found. We try to give as reference the first author known to us who explicitly gives the code and its true minimum distance.

\section{The Minimum Weight Codewords of the BCH-CODES $B\left(2^{m}-1,2^{h}-1\right)$}

We denote by $B(h), h \in[2, m-1]$, the $\mathrm{BCH}$-code of length $2^{m}-1$ and designed distance $2^{h}-1$. Since $B(h)$ contains the minimum weight codewords (mwc's) of the punctured RM-code $\mathscr{R}(m-h, m)^{*}$, its minimum distance is exactly $2^{h}-1$ (see Section II). However, the complete set of the mwc's of $B(h)$ is not known, except for the trivial cases:

$$
B(2)=\mathscr{R}(m-2, m)^{*}, \quad B(m-1)=\mathscr{R}(1, m)^{*}
$$

and

$$
B(3)=\mathscr{R}(2,5)^{*}
$$

(By identifying the defining sets). Thus, we suppose in general that

$$
h \in[3, m-2] \text { and } m>5 .
$$

In this section, we want to give some answers to the following question: Is there a mwc of $B(h)$ which is not in $\mathscr{R}(m-h, m)^{*}$ ? On the other hand, it is natural to conjecture that for each $h$, there exists a cyclic code $C \neq \mathscr{R}(m-$ $h, m)^{*}$, which is included in $B(h)$ and has for mwc's the mwc's of $\mathscr{R}(m-h, m)^{*}$.

Let $C$ be a binary cyclic code of length $n=2^{m}-1$. We denote by $M w(C)$ the set of the mwc's of $C$. We say that $C$ has the property $\left(\mathrm{RM}_{h}\right), h \in[3, m-2]$, if and only if:

$$
\begin{aligned}
\left(\mathrm{RM}_{h}\right): \quad & \mathscr{R}(m-h, m)^{*} \subset C \subseteq B(h) \text { and } \\
& M w(C)=M w\left(\mathscr{R}(m-h, m)^{*}\right),
\end{aligned}
$$

where the first inclusion is strict.

We shall prove (cf. Theorem 6) that the codes $B(m-2)$ have the property $\left(\mathrm{RM}_{m-2}\right)$. We obtain this result by exploring Newton's identities of an mwc of a code $B(h), h \in[3, m$ - 2]; we study this general case and derive the result for $h=m-2$. Moreover we can then provide an algorithm constructing cyclic codes which have the property $\left(\mathrm{RM}_{h}\right)$ for a given $h$.

Let $x$ be a mwc of $B(h)$. We have seen that $x \in \mathscr{R}(m-$ $h, m)^{*}$, if and only if its locator polynomial has the form

\begin{tabular}{|c|c|c|c|c|c|c|c|c|c|c|}
\hline$n$ & $k$ & $\delta$ & $d$ & in & $n$ & $k$ & $\delta$ & $d$ & & in \\
\hline \multirow[t]{28}{*}{511} & 502 & 3 & 3 & [7] & 511 & 241 & 73 & 73 & & [12] \\
\hline & 493 & 5 & 5 & [7] & & 238 & 75 & $\geq 75$ & & - \\
\hline & 484 & 7 & 7 & [7] & & 229 & 77 & $\geq 77$ & & - \\
\hline & 475 & 9 & 9 & $* *$ & & 220 & 79 & 79 & & $*$ \\
\hline & 466 & 11 & 11 & [7] & & 211 & 83 & 83 & & $*$ \\
\hline & 457 & 13 & 13 & [6] & & 202 & 85 & $\geq 85$ & & - \\
\hline & 448 & 15 & 15 & [7] & & 193 & 87 & $\geq 87$ & & - \\
\hline & 439 & 17 & 17 & ** & & 184 & 91 & 91 & & * \\
\hline & 430 & 19 & 19 & * & & 175 & 93 & 95 & \# & [10] \\
\hline & 421 & 21 & 21 & [12] & & 166 & 95 & 95 & & [7] \\
\hline & 412 & 23 & 23 & {$[7]$} & & 157 & 103 & 103 & & * \\
\hline & 403 & 25 & 25 & [6] & & 148 & 107 & $\geq 107$ & & - \\
\hline & 394 & 27 & 27 & [7] & & 139 & 109 & 111 & \# & [10] \\
\hline & 385 & 29 & $\geq 29$ & - & & 130 & 111 & 111 & & [7] \\
\hline & 376 & 31 & 31 & [7] & & 121 & 117 & 119 & \# & [10] \\
\hline & 367 & 35 & 35 & {$[12]$} & & 112 & 119 & 119 & & [7] \\
\hline & 358 & 37 & $\geq 37$ & - & & 103 & 123 & 127 & \# \# & $*$ \\
\hline & 349 & 39 & 39 & * & & 94 & 125 & 127 & \# & {$[10]$} \\
\hline & 340 & 41 & $\geq 41$ & - & & 85 & 127 & 127 & & [7] \\
\hline & 331 & 43 & $\geq 43$ & - & & 76 & 171 & 171 & & ** \\
\hline & 322 & 45 & 45 & * & & 67 & 175 & 175 & & $* *$ \\
\hline & 313 & 47 & 47 & [7] & & 58 & 183 & 183 & & ** \\
\hline & 304 & 51 & $\geq 51$ & - & & 49 & 187 & 187 & & $* *$ \\
\hline & 295 & 53 & 53 & * & & 40 & 191 & 191 & & [7] \\
\hline & 286 & 55 & 55 & [7] & & 31 & 219 & 219 & & [12] \\
\hline & 277 & 57 & 57 & " & & 28 & 223 & 223 & & [7] \\
\hline & 268 & 59 & $\geq 59$ & - & & 19 & 239 & 239 & & [7] \\
\hline & 259 & 61 & $\geq 61$ & - & & 10 & 255 & 255 & & [7] \\
\hline
\end{tabular}
$\sigma(Z)=\sum_{j=0}^{h} \sigma_{2^{h}-2^{j}} Z^{2^{h}-2^{j}}$ (cf. (13)). Thus, we have the following reinterpretation of the property $\left(\mathrm{RH}_{h}\right)$.
TABLE I

BCH Codes OF LeNGTH 511

$\# d=\delta+2$

$\#$ \# $d=\delta+4$

* New result obtained by Newton's identities.

${ }^{* *}$ New result obtained by an exhaustive search.

Theorem 5: For each $h \in[2, m-1]$, define:

$$
J_{h}=\left\{2^{h}-2^{j} \mid j \in[0, h]\right\} \text {. }
$$

Let $x$ be a codeword of weight $2^{h}-1$ and let $\sigma(Z)=$ $\sum_{i=0}^{2^{h}-1} \sigma_{i} Z^{i}$ be the locator polynomial of $x$. Then $\boldsymbol{x}$ is a codeword of $\mathscr{R}(m-h, m)^{*}$, if and only if $\sigma_{i}=0$ for all $i \notin J_{h}$.

Note that $0 \in J_{h}$ and that $j \in J_{h}-\{0\}$ implies $j \geq 2^{h-1}$; recall the following property of $J_{h}$ due to Kasami et al.

Lemma 3 [9]: Let $h \in[2, m-1], i_{0}=2^{h}-1$ and $r \in$ $\left[1, i_{0}[\right.$. Then,

a) $r \notin J_{h} \Rightarrow \omega_{2}\left(r+i_{0}\right)<h$.

b) $r \in J_{h} \Rightarrow \omega_{2}\left(r+i_{0}\right)=h$.

Let $S=[1, n], n=2^{m}-1$. From now on we assume that any mwc $\boldsymbol{x}$ of $\boldsymbol{B}(h)$ is defined by its locators $X_{1}, \cdots, X_{i_{0}}$. The corresponding power sum symmetric functions $A_{k}, k \in S$, and the elementary symmetric functions $\sigma_{r}$, $r \in\left[0, i_{0}\right]$ are related by the Newton's identities $I_{k}, k \in S$. By definition $\sigma_{0}=1$; since $x \in B(h)$ we know that

- $A_{k}=0$, for $k \in\left[1, i_{0}[\right.$,

- $r$ odd and $r<i_{0} \Rightarrow \sigma_{r}=0$,

- $A_{i_{0}}$ cannot be zero, since the minimum distance of $B(h)$ is exactly $i_{0}$.

Then identities $I_{k}$ are satisfied for $k<i_{0}$; identity $I_{i_{0}}$ yields $\sigma_{i_{0}}=A_{i_{0}}$. In accordance with Theorem 5 , we shall study the 
following hypothesis $H_{r}$ :

$$
H_{r}: r \in\left[1, i_{0}\left[\text { and } r \notin J_{h} \Rightarrow \sigma_{r}=0 \text { and } A_{i_{0}+r}=0\right. \text {. }\right.
$$

We know that $H_{r}$ is true for $r$ odd (cf. Lemma 1). Recall the form of identity $I_{i_{0}+r}$ :

$$
I_{i_{0}+r}: A_{i_{0}+r}+\sum_{k=1}^{r} A_{i_{0}+r-k} \sigma_{k}=0 .
$$

The following lemma means that the code $B(h)$ has the property $\left(\mathrm{RM}_{\mathrm{h}}\right)$, if and only if $H_{r}$ is true for all $r \in\left[2, i_{0}[\right.$, $i_{0}=2^{h}-1$. This result still holds for any cyclic code $C$ which contains $\mathscr{R}(m-h, m)^{*}$ and is contained in $B(h)$. We now proceed by induction on $r$ to show that $H_{r}$ is true.

Lemma 4: Let $r$ be even. Suppose that $H_{r^{\prime}}$ is true for all $r^{\prime} \in[1, r[$. Then we have

$$
I_{i_{0}+r}: A_{i_{0}+r}+A_{i_{0}} \sigma_{r}=0 .
$$
$k \in[1, r[:$

Proof: We examine the term $A_{i_{0}+r-k} \sigma_{k}$ in (15), for

- if $k \notin J_{h}$ then $H_{k}$ implies $\sigma_{k}=0$;

- if $k \in J_{h}$ then $k \geq 2^{h-1}$; hence $r-k<2^{h-1}$, which means that $r-k$ is not in $J_{h}$; applying $H_{r-k}$, we obtain $A_{i_{0}+r-k}=0$.

Remark: We know that the locator polynomials of the mwc's of $\mathscr{R}(m-h, m)^{*}$ satisfy $\sigma_{i}=0$ for $i \notin J_{h}$. From Lemma 4, we obtain another property:

$$
r \in J_{h} \Rightarrow \sigma_{r}=\frac{A_{i_{0}+r}}{A_{i_{0}}} .
$$

Example 1: The BCH-codes of designed distance 7, i.e., $h=3, i_{0}=7$, and $J_{3}=\{0,4,6,7\}$. Recall that the defining-sets of $\mathscr{R}(m-3, m)^{*}$ and $B(3)$ are

$$
\begin{gathered}
S_{3}=\left\{s \in S \mid \omega_{2}(s)<3\right\} \quad \text { and } \\
I(B(3))=\operatorname{cl}(1) \cup \operatorname{cl}(3) \cup \operatorname{cl}(5),
\end{gathered}
$$

respectively. Since $\sigma_{r}=0$ for $r$ odd, Lemma 1 implies that $B(3)$ has the property $\left(\mathrm{RM}_{3}\right)$ if and only if $\sigma_{2}=0$; we have seen that $H_{1}$ is always true; from Lemma $4, \sigma_{2}=0$ if and only if $A_{9}=0$. In other words: $B(3)$ has the property $\left(\mathrm{RM}_{3}\right)$ if and only if each mwc of $B(3)$ is such that $A_{9}=0$. We conjecture that, in general, $B(3)$ does not have the property $\left(\mathrm{RM}_{3}\right)$. For $m \in\{6,7,8,9\}$, we have obtained (with a computer) a mwc of $B(3)$ which is not in $\mathscr{R}(m-$ $3, m)^{*}$.

Let $\hat{T}=\operatorname{cl}(9) \cup I(B(3))$. Since $\omega_{2}(9)=2, \hat{T} \subset S_{3}$. Now, we examine a code $C$ whose defining-set $T$ is such that $\hat{T} \subseteq T \subset S_{3}$, where the right inclusion is strict. Thus $C$ contains $\mathscr{R}(m-3, m)^{*}$ and is contained in $B(3)$. Moreover each codeword of $C$ is such that its power sum symmetric function $A_{9}$ equals zero. If $m \in\{6,7\}$, it is easy to see that $\hat{T}$ equals $S_{3}$. When $m>7,17$ is in $S_{3}$ and not in $\hat{T}$. In conclusion, we have the following.

1) Assume that $m>7$. Then a cyclic code $C$ with defining-set $T$ satisfying:

$c l(1) \cup \operatorname{cl}(3) \cup \operatorname{cl}(5) \cup \operatorname{cl}(9) \subseteq T \subset\left\{s \in S \mid \omega_{2}(s)<3\right\}$ has the property $\left(\mathrm{RM}_{3}\right)$. Conversely, we conjecture that a code $C$ which has the property $\left(\mathrm{RM}_{3}\right)$, satisfies the above property.

2) If $m \leq 7$, it is impossible to construct a code $C$ which has the property $\left(\mathrm{RM}_{3}\right)$.

Now we will distinguish the two cases: $r \leq 2^{h-1}$ and $r>2^{h-1}$.

Lemma 5: Assume that $r \in\left[2,2^{h-1}\right], r$ even, and that $H_{r^{\prime}}$ is true for all $r^{\prime}<r$. Then identity $I_{2 i_{0}+3 r}$ becomes

$I_{2 i_{0}+3 r}: A_{2 i_{0}+3 r}+A_{i_{0}+r}^{2} \sigma_{r}+A_{i_{0}+3 r} \sigma_{i_{0}}=0$,

$$
\text { if } r>\frac{i_{0}-1}{3}
$$

and

$$
\begin{array}{r}
I_{2 i_{0}+3 r}: A_{2 i_{0}+3 r}+A_{i_{0}+r}^{2} \sigma_{r}+A_{i_{0}}^{2} \sigma_{3 r}+A_{i_{0}+3 r} \sigma_{i_{0}}=0, \\
\text { if } r \leq \frac{i_{0}-1}{3} .
\end{array}
$$

Proof: $i_{0}=2^{h}-1$. Note that $2 i_{0}+3 r<2^{h+1}+$ $3.2^{h-1}<2^{m}-1$, since $h<m-1$. Then the identity $I_{2 i_{0}+3 r}$ is defined. Its general form is

$$
I_{2 i_{0}+3 r}: A_{2 i_{0}+3 r}+\sum_{k=1}^{i_{0}-1} A_{2 i_{0}+3 r-k} \sigma_{k}+A_{i_{0}+3 r} \sigma_{i_{0}}=0 .
$$

Suppose that $H_{r^{\prime}}$ is true for $r^{\prime}<r$ and consider the term $A_{2 i_{0}+3 r-k} \sigma_{k}$. If $k$ is odd, then $\sigma_{k}=0$. If $k$ is even, let $r-k=2 k^{\prime}$; we have

$$
A_{2\left(i_{0}+r\right)+r-k} \sigma_{k}^{\prime}=A_{i_{0}+r+k^{\prime}}^{2} \sigma_{k}, \quad k \in\left[1, i_{0}[.\right.
$$

Then,

- $k^{\prime}>0 \Rightarrow k<r \Rightarrow \sigma_{k}=0$, from $H_{k}$ ( $k$ cannot be in $J_{h}$, since $r \leq 2^{h-1}$ ).

- $k^{\prime}<0$ and $r+k^{\prime} \neq 0 \Rightarrow r+k^{\prime}<r \Rightarrow A_{i_{0}+r+k^{\prime}}=0$ (if $r+k^{\prime}>0$ apply $H_{r+k^{\prime}}$ otherwise $i_{0}+r+k^{\prime}$ is an element of the defining set of $B(h)$ ).

- $r+k^{\prime}=0$ is obtained when it is possible to have $k=3 r$; since $k<i_{0}$, this condition implies $r \leq\left(i_{0}-\right.$ 1) $/ 3$.

In conclusion, identity $I_{2 i_{0}+3 r}$ reduces to (17) if $r>\left(i_{0}-\right.$ 1) $/ 3$ and to (18) otherwise.

Lemma 6: $r$ even and $i_{0}=2^{h}-1, h \in[3, m-2]$. Assume that $r \in\left[2^{h-1}, i_{0}\left[\right.\right.$ and $r \notin J_{h}$; suppose that $H_{r^{\prime}}$ is true for all $r^{\prime}<r$. Then identity $I_{2\left(i_{0}+r\right)}$ becomes

$$
I_{2\left(i_{0}+r\right)}: A_{i_{0}+r}^{2}+A_{i_{0}+2 r} \sigma_{i_{0}}=0 .
$$

Proof: Note that $2 i_{0}+2 r \leq 4 i_{0}-2 \leq 2^{h+2}-6<$ $2^{m}-1$. Hence, an identity $I_{2 i_{0}+2 r}$ is defined. Its general form is

$I_{2\left(i_{0}+r\right)}: A_{i_{0}+r}^{2}+\sum_{k=1}^{i_{0}-1} A_{2\left(i_{0}+r\right)-k} \sigma_{k}+A_{i_{0}+2 r} \sigma_{i_{0}}=0$.

Suppose that $H_{r^{\prime}}$ is true for $r^{\prime}<r$; consider for $k$ even, the general term $A_{i_{0}+r-k^{\prime}}^{2} \sigma_{k}$, where $k=2 k^{\prime}$. Notice that, by 
hypothesis,

$$
k^{\prime}<\frac{i_{0}}{2} \Rightarrow r-k^{\prime}>2^{h-1}-\frac{2^{h}-1}{2} \Rightarrow 0<r-k^{\prime}<r .
$$

Hence, if $r-k^{\prime} \notin J_{h}$ then $A_{i_{0}+r-k^{\prime}}=0$ (from $H_{r-k^{\prime}}$ ). Suppose that $r-k^{\prime} \in J_{h}$; then there is an integer $j \in[1, h-$ 1] such that $r-k^{\prime}=2^{h}-2^{j}$. Now we have two possibilities.

1) $2 k^{\prime}<r$. If $k \notin J_{h}$ then $\sigma_{k}=0$; if $k \in J_{h}$ there is a $j^{\prime} \in[1, h-1]$ such that $k=2^{h}-2^{j^{\prime}}$; thus, $r=2^{h}$ $+2^{h-1}-2^{j}-2^{j^{\prime}-1}$; if $j<h-1$ we obtain $r>2^{h}$ -1 ; if $j=h-1$ we obtain $r \in J_{h}$; so in all cases we have a contradiction to the hypothesis on $r$.

2) $2 k^{\prime} \geq r \Rightarrow r-k^{\prime} \leq k^{\prime} \leq\left(2^{h}-2\right) / 2$. Then $r-k^{\prime}$ cannot be in $J_{h}$; that contradicts the hypothesis on $r-k^{\prime}$.

Lemma 7: Assume that $h=m-2$. Recall that the defining-set of $B(m-2)$ is denoted by $I(B(m-2))$ and that $i_{0}=2^{h}-1$. The following properties are satisfied.

a) Let $s \in\left[0,2^{m}-1\right]$ and let $\sum_{k=0}^{m-1} s_{k} 2^{k}$ be the 2-ary expansion of $s$. Then $s \in I(B(h))$, if and only if there is $k<m$ and $j \notin\left\{k, k^{\prime}=k+1(\bmod m)\right\}$ such that $s_{k}=s_{k^{\prime}}=s_{j}=0$.

b) Let $r \in\left[2,2^{h-1}\left[, r\right.\right.$ even such that $i_{0}+r \notin I(B(h))$. Then

$$
2 i_{0}+3 r \in I(B(h)) \text { and } i_{0}+3 r \in I(B(h)) .
$$

c) Let $r \in] 2^{h-1}, i_{0}\left[, r\right.$ even and $r \notin J_{h}$. Then there is an element of the cyclotomic class of $i_{0}+2 r$ (modulo $2^{m}-1$ ), which can be written as

$$
i_{0}+\epsilon \quad \text { with }-i_{0}<\epsilon<r \text { and } \epsilon \notin J_{h} .
$$

Proof: a) The hypotheses on $s$ mean that $2^{m-k-1} s<$ $2^{m-2}-1$, i.e., that $s$ is an element of the defining-set of $B(m-2)$.

b) Let $r^{\prime}=r / 2$. By hypothesis, the 2-ary expansion of $i_{0}, r$ and $r^{\prime}$ are

$$
i_{0}=\sum_{j=0}^{m-3} 2^{j}, \quad r=\sum_{j=1}^{m-4} r_{j} 2^{j}, \quad r^{\prime}=\sum_{j=0}^{m-5} r_{j+1} 2^{j} .
$$

Note that $i_{0}+r+r^{\prime}<2^{m-2}+3.2^{m-4}-1<2^{m-1}$. Let $k$ be the smallest $j$ such that $r_{j} \neq 0$. Suppose that $r_{k+1}=0$ or that $k=m-4$. We have

$$
\begin{aligned}
i_{0}+r=\sum_{j=0}^{k-1} 2^{j}+(1+1) 2^{k} & +2^{k+1} \\
& +\sum_{j=k+2}^{m-4}\left(1+r_{j}\right) 2^{j}+2^{m-3} .
\end{aligned}
$$

Then the 2-ary expansion of $i_{0}+r$ is such that its $k$ th term and its $(k+1)$ th term are zero. From a), that means $i_{0}+r$ $\in I(B(h))$, which contradicts the hypothesis. Thus $r_{k+1}=1$ and $k<m-4$. Now the 2-ary expansion of $i_{0}+r+r^{\prime}$ is

$$
\begin{aligned}
i_{0}+r+r^{\prime}= & \sum_{j=0}^{k-2} 2^{j}+(1+0+1) 2^{k-1}+(1+1+1) 2^{k} \\
& +\sum_{j=k+1}^{m-5}\left(1+r_{j}+r_{j+1}\right) 2^{j} \\
& +\left(1+r_{m-4}\right) 2^{m-4}+2^{m-3}
\end{aligned}
$$

(by convention a sum from $a$ to $b$, with $a>b$, equals 0 ). So we can see that the $(k-1)$ th term and the $k$ th term are zero. We can apply a): the defining set of $B(h)$ contains $2\left(i_{0}+r+r^{\prime}\right)=2 i_{0}+3 r$. Now we have

$i_{0}+3 r<2^{m-2}-1+3.2^{m-3}$

$$
=2^{m-1}+2^{m-3}-1<2^{m}-1 \text {. }
$$

We consider the 2-ary expansion of $i_{0}+3 r$ :

$$
\begin{aligned}
i_{0}+r+2 r= & \sum_{j=0}^{k-1} 2^{j}+(1+1+0) 2^{k} \\
& +(1+1+1) 2^{k+1} \\
& +\sum_{j=k+2}^{m-4}\left(1+r_{j}+r_{j-1}\right) 2^{j} \\
& +\left(1+r_{m-4}\right) 2^{m-3}
\end{aligned}
$$

The $k$ th term and the $(k+1)$ th term are zero. Moreover, from (22), an $l$ th term, $l \in\{m-1, m-2, m-3\}$ is zero. Applying a), we obtain $i_{0}+3 r \in I(B(h))$.

c) By hypothesis, $2^{m-1}-1<i_{0}+2 r<2^{m}-1$. We consider another element of the cyclotomic class of $i_{0}+2 r$ :

$$
2\left(i_{0}+2 r\right)-\left(2^{m}-1\right)=i_{0}+\epsilon,
$$

where

$$
\epsilon=4 r+2^{m-2}-2^{m} \text {. }
$$

Since $r$ is even and $2^{m-3}<r<2^{m-2}$, we have

$$
\epsilon>4\left(2^{m-3}+1\right)+2^{m-2}-2^{m} \Rightarrow \epsilon>4-2^{m-2}>-i_{0}
$$

and

$$
\epsilon=r+3\left(r-2^{m-2}\right) \Rightarrow \epsilon<r-3 .
$$

Suppose that $\epsilon \in J_{h}$. Then there is a $j \in[0, m-2]$ such that

$$
4 r+2^{m-2}-2^{m}=2^{m-2}-2^{j} \Rightarrow r=2^{m-2}-2^{j-2},
$$

which implies $r \in J_{h}$, contradicting the hypothesis. We have proved that $\epsilon$ cannot be in $J_{h}$.

Now we are able to prove that the code $B(m-2)$ has the property $\left(\mathrm{RM}_{m-2}\right)$.

Theorem 6: The minimum weight codewords of the BCH-codes of length $2^{m}-1$ and designed distance $2^{m-2}-1$ are those of the punctured RM-code of the same length and order 2 .

Proof: The notations are as previously defined; moreover assume that $h=m-2$. We shall prove that, for this particular value of $h, H_{r}$ is true for all $r \in\left[1, i_{0}[\right.$. If $r$ is 
odd, we know that $H_{r}$ is true; we suppose that $H_{r}$ is true for all $r^{\prime} \in\left[2, r\right.$ [ and we want to prove that $H_{r}$ is true.

If $i_{0}+r \in I(B(h))$ then $A_{i_{0}+r}=0$ (by definition of $B(h)) ; A_{i_{0}}$ cannot be zero, since the designed distance $i_{0}$ of $B(h)$ is exactly its minimum distance. Thus, Lemma 4 implies $\sigma_{r}=0$; then $H_{r}$ is true. So we suppose now that $r$ is even and that $i_{0}+r \notin I(B(h))$. We consider two cases.

1) Assume that $r \in\left[2,2^{h-1}\right.$ [. Then $r$ cannot be in $J_{h}$. Let $\rho$ be the smallest element of $\left[i_{0}+1,2 i_{0}[\right.$, such that $\rho \notin I(B(m-2))$. From Lemma $7 \mathrm{a})$, we have

$$
\rho=\left\{\begin{array}{cl}
\sum_{k=0}^{(m-2) / 2} 2^{2 k}=\left(2^{m}-1\right) / 3, & \text { if } m \text { is even, } \\
1+\sum_{k=0}^{(m-3) / 2} 2^{2 k+1} & \\
=1+2\left(2^{m-1}-1\right) / 3, & \text { if } m \text { is odd. }
\end{array}\right.
$$

If we suppose that $i_{0}+r \notin I(B(m-2))$, then $i_{0}+r$ $\geq \rho$. If $m$ is even then

$$
\rho-i_{0}=\frac{2^{m}-1}{3}-2^{m-2}+1=\frac{2^{m-2}+2}{3},
$$

and if $m$ is odd then

$$
\begin{aligned}
\rho-i_{0} & =1+2 \frac{2^{m-1}-1}{3}-2^{m-2}+1 \\
& =1+\frac{2^{m-2}+1}{3} .
\end{aligned}
$$

Hence, in all cases, $r>\left(i_{0}-1\right) / 3$. From Lemma 5, the identity $I_{2 i_{0}+3 r}$ is reduced to (17). From Lemma 7b), $A_{2 i_{0}+3 r}=A_{i_{0}+3 r}=0$. Then

$I_{2 i_{0}+3 r}: A_{i_{0}+r}^{2} \sigma_{r}=0$ and $I_{i_{0}+r}: A_{i_{0}+r}+A_{i_{0}} \sigma_{r}=0$, which yields $A_{i_{0}+r}=0$ and $\sigma_{r}=0$, i.e., $H_{r}$ is true.

2) Assume that $r \in\left[2^{h-1}, i_{0}\left[\right.\right.$. If $r \in J_{h}$ then $H_{r}$ is true. So we suppose that $r \notin J_{h}$. From Lemma 7c), there is an $\epsilon,-i_{0}<\epsilon<r$ and $\epsilon \notin J_{h}$, such that $i_{0}+\epsilon$ is an element of the cyclotomic class of $i_{0}+2 r$; thus,

- $-i_{0}<\epsilon<0 \Rightarrow i_{0}+\epsilon \in I(B(h)) \Rightarrow A_{i_{0}+\epsilon}=0 \Rightarrow$ $A_{i_{0}+2 r}=0$,

- $0<\epsilon<r \Rightarrow A_{i_{0}+\epsilon}=0$, (from $H_{\epsilon}$ ) $\Rightarrow A_{i_{0}+2 r}=0$.

From Lemma 6, the identity $\left.I_{2\left(i_{0}+r\right.}\right)$ reduces to $A_{i_{0}+r}^{2}=0$. From Lemma 4 , this yields $\sigma_{r}=0$, i.e., $H_{r}$ is true. In accordance with Theorem 5 , we have proved that $B(m-2)$ has the property $\left(\mathrm{RM}_{m-2}\right)$. Remark that $\mathscr{R}(2, m)^{*}$ is strictly contained in the code $B(m-2)$; for instance $2^{m-1}-2^{g+1}$ $+2^{g}-1$, for $0<g<m-1$, is in the defining set of $\mathscr{R}(2, m)^{*}$ but not in $I(B(m-2))$.

Let $x \in B(m-2)$ such that $\omega(x)=2^{m-2}=\mu$; let $X=$ $\left\{X_{1}, \cdots, X_{\mu}\right\}$ be the set of locators of $x$. It is well known that the extended $\mathrm{BCH}$-codes and the Reed-Muller codes are invariant under the affine group ([8], [2]); this means that, for each $g \in \mathrm{GF}\left(2^{m}\right)$, the locators $\left\{X_{1}+g, \cdots, X_{\mu}+g\right\}$ are those of a codeword in the extension of the code $B(m-$ 2). In particular, we can state

$$
\begin{gathered}
X=X_{1}+\{0\} \cup X^{\prime}, \\
X^{\prime}=\left\{X_{2}+X_{1}, \cdots, X_{\mu}+X_{1}\right\},
\end{gathered}
$$

where $X^{\prime}$ is the set of the locators of a codeword $\boldsymbol{x}^{\prime}$ of $B(m-2)$. Moreover $\boldsymbol{x}^{\prime}$ is a mwc of $B(m-2)$. Hence, Theorem 6 implies the following corollary.

Corollary 1: Let $x \in B(m-2)$ be such that $\omega(x)=$ $2^{m-2}$. Then $x$ is a codeword of the punctured RM-code $\mathscr{R}(2, m)^{*}$, i.e., the set of the locators of $x$ is an $(m-2)$ dimensional affine subspace of GF $\left(2^{m}\right)$.

It is well known that the automorphism group of the binary punctured Reed-Muller codes is the general linear group, denoted by GL $(2, m)([11]$, p. 400$)$; moreover the punctured RM-codes are generated by their mwc's ([11, p. 381]). Hence, a code $C$ which has the property $\left(\mathrm{RM}_{h}\right)$ is such that its automorphism group is contained in $\operatorname{GL}(2, m)$. Moreover, such a code cannot be generated by $M w(C)$, since $\mathscr{R}(m-$ $h, m)^{*}$ is strictly contained in it.

Corollary 2: $m>5$. The automorphism group of the $\mathrm{BCH}$-code $B(m-2)$ is contained in $G L(2, m)$. The code generated by the set of the minimum weight codewords of $B(m-2)$ is strictly contained in $B(m-2)$.

The property $\left(\mathrm{RM}_{3}\right)$ is studied in Example 1, and Theorem 6 gives a general result for the property $\left(\mathrm{RM}_{m-2}\right)$. From now on, we are interested in the definition of cyclic codes which have the property $\left(\mathrm{RM}_{h}\right)$, for $h \in[4, m-3], m>6$. We study the property $\left(\mathrm{RM}_{h}\right)$ by explaining the hypotheses on the mwc's of the codes $B(h)$. The main idea is that Newton's identities yield certain conditions on the power sum symmetric functions of these codewords. In accordance with Theorem 5 and Lemma 4, we can state a sufficient condition for a cyclic code to have the property $\left(\mathrm{RM}_{h}\right)$.

Corollary 3: Let us define

$$
\begin{gathered}
T_{h}=\bigcup_{s \in U_{h}} c l(s), \\
U_{h}=\left\{s \in \left[i_{0}+1,2 i_{0}\left[\mid s \notin I(B(h)), \omega_{2}(s)<h\right\} .\right.\right.
\end{gathered}
$$

Let $C$ be a cyclic code such that its defining set $T$ satisfies

$$
I(B(h)) \cup T_{h} \subseteq T \subseteq\left\{s \in S \mid \omega_{2}(s)<h\right\}
$$

If $\mathscr{R}(m-h, m)^{*}$ is strictly contained in $C$, then $C$ has the property $\left(\mathrm{RM}_{h}\right)$.

Proof: Suppose that $C \neq \mathscr{R}(m-h, m)^{*}$. Then the second inclusion in (24) is strict. From Lemma 3 , the elements of $T_{h}$ are of the form $s=i_{0}+r$ with $r \notin J_{h}$. Then any mwc of $C$ is a mwc of $B(h)$ which satisfies $A_{i_{0}+r}=0$ for all $r \notin J_{h}$ (with $r \in\left[1, i_{0}[\right.$ ). Applying Lemma 4, we prove by induction that $H_{r}$ is true for all $r$. Then $C$ has the property $\left(\mathrm{RM}_{h}\right)$.

The following conjectures are reinforced by results we 
have obtained with a computer. For $h \in[4, m-3], m>6$ :

1) the codes $B(h)$ do not have the property $\left(\mathrm{RM}_{h}\right)$,

2) there exists a cyclic code $C$, the definition set of which is strictly contained in $I(B(h)) \cup T_{h}$, which has the property $\left(\mathrm{RM}_{h}\right)$.

We give later some examples which prove that the second conjecture is true for $m=7$ and $m=8$. We use the fact that the proof of Theorem 6, applied to the general case $h \neq m-2$, provides an algorithm constructing a cyclic code which has the property $\left(\mathrm{RM}_{h}\right)$, for a given $m$. In the following, $C$ is a cyclic code such that $\mathscr{R}(m-h, m)^{*} \subset C$ $\subset B(h) ; T$ denotes its defining set. The proof of the proposed algorithm is obvious: using the results of Lemmas 4 , 5 , and 6 , we construct $T$ such that $H_{r}$ is true for all $r \in\left[1, i_{0}\right)[$; if $r$ is such that Newton's identities, given by (17) or (18) or (20), do not imply $A_{i_{0}+r}=0$, then we add $i_{0}+r$ in $T$.

\section{Algorithm Constructing $T$}

1) $T=I(B(h)) ; r=0 ; i_{0}=2^{h}-1$;

2) $r=r+2$; IF $r>i_{0}$ THEN go to 8 );

3) IF $i_{0}+r \in T$ THEN put $\sigma_{r}:=0$ and Go To 2);

4) IF $r<2^{h-1}$, examine the identity $I_{2 i_{0}+3 r}$ :

IF $I_{2 i_{0}+3 r}: A_{i_{0}+r}^{2} \sigma_{r}=0$ THEN GO TO 7 )

ELSE PUT $T:=T \cup \mathrm{cl}\left(i_{0}+r\right)$ and GO TO 7);

5) IF $r \in J_{h}$ THEN GO TO 2);

6) IF $r>2^{h-1}$, examine the identity $I_{2\left(i_{0}+r\right)}$ :

IF $I_{2\left(i_{0}+r\right)}: A_{i_{0}+r}^{2}=0$ THEN GO TO 7$)$

ELSE PUT $T:=T \cup \operatorname{cl}\left(i_{0}+r\right)$;

7) PUT $\sigma_{r}:=0$ and $A_{i_{0}+r}^{2^{j}}:=0$, for $j \in[0, m-1]$; GO To 2);

8) END.

Example 2: $m=7 ; h=4$; thus $i_{0}=15$ and $J_{4}=$ $\{0,8,12,14\}$. The code $B(h)$ is the BCH-code of length 127 and designed distance 15 . In accordance with Corollary 3 , we have $T_{4}=c l(19) \cup \operatorname{cl}(21)$. Using the algorithm, we obtain that the code $C$ with defining set $T=I(B(4)) \cup \operatorname{cl}(19)$, satisfies $\left(\mathrm{RM}_{4}\right)$

Example 3: $m=8$.

1) $h=4$. The code $B(4)$ is the $\mathrm{BCH}$-code of length 255 and designed distance 15 . We have

$$
T_{4}=c l(17) \cup c l(19) \cup \operatorname{cl}(21) \cup \operatorname{cl}(25) \text {. }
$$

The algorithm produces: $T=I(B(4)) \cup \operatorname{cl}(17) \cup$ cl(19).

2) $h=5 ; J_{5}=\{0,16,24,28,30,31\}$. The code $B(5)$ is the $\mathrm{BCH}$-code of length 255 and designed distance 31 . We have

$$
\begin{gathered}
T_{5}=c l(37) \cup \operatorname{cl}(39) \cup \operatorname{cl}(43) \cup \operatorname{cl}(45) \\
\cup c l(51) \cup c l(53) .
\end{gathered}
$$

The algorithm produces: $T=I(B(5)) \cup \operatorname{cl}(37) \cup$ $\operatorname{cl}(39)$.
APPENDIX A

$B(255,61)$ has Minimum Distance $>61$

We consider Newton's identities $I_{r}$ for $0<r \leq n=255$, for the code $B(255,61)$, and for the weight $\delta=61$. We want to prove that there exists no codeword of weight $\delta$.

The nonzero power sum symmetric functions of the code are

$$
A_{61}, A_{63}, A_{85}, A_{87}, A_{91}, A_{95}, A_{111}, A_{119}, A_{127} \text {. }
$$

Since 255 and 61 are relatively prime we can suppose $A_{61}=1$ (the shift corresponds to a multiplication of each $A_{i}$ by $\alpha^{i}$ ).

In the case of a narrow-sense primitive $\mathrm{BCH}$ code, and for a weight equal to the designed distance, Newton's identities $I_{r}(9)$ for odd $r$ from $\delta+2$ to $2 \delta-1$ form a triangular linear system giving the $\sigma_{i}$ 's for even $i$ as polynomials depending on the nonzero $A_{i}$ 's. Here the system consists of the following 30 equations:

$$
\begin{aligned}
& I_{63}: A_{63}+\sigma_{2}=0 \\
& I_{65}: A_{63} \sigma_{2}+\sigma_{4}=0 \\
& I_{67}: A_{63} \sigma_{4}+\sigma_{6}=0 \\
& I_{69}: A_{63} \sigma_{6}+\sigma_{8}=0 \\
& I_{71}: A_{63} \sigma_{8}+\sigma_{10}=0 \\
& I_{73}: A_{63} \sigma_{10}+\sigma_{12}=0 \\
& I_{75}: A_{63} \sigma_{12}+\sigma_{14}=0 \\
& I_{77}: A_{63} \sigma_{14}+\sigma_{16}=0 \\
& I_{79}: 1+A_{63} \sigma_{16}+\sigma_{18}=0 \\
& I_{81}: \sigma_{2}+A_{63} \sigma_{18}+\sigma_{20}=0 \\
& I_{83}: \sigma_{4}+A_{63} \sigma_{20}+\sigma_{22}=0 \\
& I_{85}: A_{85}+\sigma_{6}+A_{63} \sigma_{22}+\sigma_{24}=0 \\
& I_{87}: A_{87}+A_{85} \sigma_{2}+\sigma_{8}+A_{63} \sigma_{24}+\sigma_{26}=0 \\
& I_{89}: A_{87} \sigma_{2}+A_{85} \sigma_{4}+\sigma_{10}+A_{63} \sigma_{26}+\sigma_{28}=0 \\
& I_{91}: A_{91}+A_{87} \sigma_{4}+A_{85} \sigma_{6}+\sigma_{12}+A_{63} \sigma_{28}+\sigma_{30}=0 \\
& I_{93}: A_{87}^{4}+A_{91} \sigma_{2}+A_{87} \sigma_{6}+A_{85} \sigma_{8}+\sigma_{14}+A_{63} \sigma_{30}+\sigma_{32}=0 \\
& I_{95}: A_{95}+A_{87}^{4} \sigma_{2}+A_{91} \sigma_{4}+A_{87} \sigma_{8}+A_{85} \sigma_{10}+\sigma_{16} \\
& +A_{63} \sigma_{32}+\sigma_{34}=0 \\
& I_{97}: A_{95} \sigma_{2}+A_{87}^{4} \sigma_{4}+A_{91} \sigma_{6}+A_{87} \sigma_{10}+A_{85} \sigma_{12}+\sigma_{18} \\
& +A_{63} \sigma_{34}+\sigma_{36}=0 \\
& I_{99}: A_{95} \sigma_{4}+A_{87}^{4} \sigma_{6}+A_{91} \sigma_{8}+A_{87} \sigma_{12}+A_{85} \sigma_{14}+\sigma_{20} \\
& +A_{63} \sigma_{36}+\sigma_{38}=0 \\
& I_{101}: A_{95} \sigma_{6}+A_{87}^{4} \sigma_{8}+A_{91} \sigma_{10}+A_{87} \sigma_{14}+A_{85} \sigma_{16}+\sigma_{22} \\
& +A_{63} \sigma_{38}+\sigma_{40}=0 \\
& I_{103}: A_{95} \sigma_{8}+A_{87}^{4} \sigma_{10}+A_{91} \sigma_{12}+A_{87} \sigma_{16}+A_{85} \sigma_{18}+\sigma_{24} \\
& +A_{63} \sigma_{40}+\sigma_{42}=0 \\
& I_{105}: A_{95} \sigma_{10}+A_{87}^{4} \sigma_{12}+A_{91} \sigma_{14}+A_{87} \sigma_{18}+A_{85} \sigma_{20}+\sigma_{26} \\
& +A_{63} \sigma_{42}+\sigma_{44}=0
\end{aligned}
$$




$$
\begin{gathered}
I_{107}: A_{91}^{32}+A_{95} \sigma_{12}+A_{87}^{4} \sigma_{14}+A_{91} \sigma_{16}+A_{87} \sigma_{20} \\
+A_{85} \sigma_{22}+\sigma_{28}+A_{63} \sigma_{44}+\sigma_{46}=0 \\
I_{109}: A_{91}^{4}+A_{91}^{32} \sigma_{2}+A_{95} \sigma_{14}+A_{87}^{4} \sigma_{16}+A_{91} \sigma_{18}+A_{87} \sigma_{22} \\
+A_{85} \sigma_{24}+\sigma_{30}+A_{63} \sigma_{46}+\sigma_{48}=0 \\
I_{111}: A_{111}+A_{91}^{4} \sigma_{2}+A_{91}^{32} \sigma_{4}+A_{95} \sigma_{16}+A_{87}^{4} \sigma_{18}+A_{91} \sigma_{20} \\
+A_{87} \sigma_{24}+A_{85} \sigma_{26}+\sigma_{32}+A_{63} \sigma_{48}+\sigma_{50}=0 \\
I_{113}: A_{111} \sigma_{2}+A_{91}^{4} \sigma_{4}+A_{91}^{32} \sigma_{6}+A_{95} \sigma_{18}+A_{87}^{4} \sigma_{20}+A_{91} \sigma_{22} \\
+A_{87} \sigma_{26}+A_{85} \sigma_{28}+\sigma_{34}+A_{63} \sigma_{50}+\sigma_{52}=0 \\
I_{115}: A_{111} \sigma_{4}+A_{91}^{4} \sigma_{6}+A_{91}^{32} \sigma_{8}+A_{95} \sigma_{20}+A_{87}^{4} \sigma_{22}+A_{91} \sigma_{24} \\
+A_{87} \sigma_{28}+A_{85} \sigma_{30}+\sigma_{36}+A_{63} \sigma_{52}+\sigma_{54}=0 \\
I_{117}: A_{87}^{16}+A_{111} \sigma_{6}+A_{91}^{4} \sigma_{8}+A_{91}^{32} \sigma_{10}+A_{95} \sigma_{22}+A_{87}^{4} \sigma_{24} \\
+A_{91} \sigma_{26}+A_{87} \sigma_{30}+A_{85} \sigma_{32} \\
+\sigma_{38}+A_{63} \sigma_{54}+\sigma_{56}=0 \\
I_{119}: A_{119}+A_{87}^{16} \sigma_{2}+A_{111} \sigma_{8}+A_{91}^{4} \sigma_{10}+A_{91}^{32} \sigma_{12}+A_{95} \sigma_{24} \\
+A_{87}^{4} \sigma_{26}+A_{91} \sigma_{28}+A_{87} \sigma_{32} \\
+A_{85} \sigma_{34}+\sigma_{40}+A_{63} \sigma_{56}+\sigma_{58}=0 \\
I_{121}: A_{119} \sigma_{2}+A_{87}^{16} \sigma_{4}+A_{111} \sigma_{10}+A_{91}^{4} \sigma_{12}+A_{91}^{32} \sigma_{14} \\
+A_{95} \sigma_{26}+A_{87}^{4} \sigma_{28}+A_{91} \sigma_{30}+A_{87} \sigma_{34} \\
+A_{85} \sigma_{36}+\sigma_{42}+A_{63} \sigma_{58}+\sigma_{60}=0 \\
\end{gathered}
$$

which gives us the following values for the $\sigma_{i}$ 's:

$$
\begin{aligned}
& \sigma_{2}:=A_{63} \\
& \sigma_{4}:=A_{63}^{2} \\
& \sigma_{6}:=A_{63}^{3} \\
& \sigma_{8}:=A_{63}^{4} \\
& \sigma_{10}:=A_{63}^{5} \\
& \sigma_{12}:=A_{63}^{6} \\
& \sigma_{14}:=A_{63}^{7} \\
& \sigma_{16}:=A_{63}^{8} \\
& \sigma_{18}:=1+A_{63}^{9} \\
& \sigma_{20}:=A_{63}^{10} \\
& \sigma_{22}:=A_{63}^{2}+A_{63}^{11} \\
& \sigma_{24}:=A_{85}+A_{63}^{12} \\
& \sigma_{26}:=A_{87}+A_{63}^{4}+A_{63}^{13} \\
& \sigma_{28}:=A_{85} A_{63}^{2}+A_{63}^{14} \\
& \sigma_{30}:=A_{91}+A_{87} A_{63}^{2}+A_{63}^{6}+A_{63}^{15} \\
& \sigma_{32}:=A_{87}^{4}+A_{85} A_{63}^{4}+A_{63}^{16} \\
& \sigma_{34}:=A_{95}+A_{91} A_{63}^{2}+A_{87} A_{63}^{4}+A_{63}^{8}+A_{63}^{17} \\
& \sigma_{36}:=A_{87}^{4} A_{63}^{2}+A_{85} A_{63}^{6}+1+A_{63}^{18}
\end{aligned}
$$

$$
\begin{aligned}
\sigma_{38}:=A_{95} A_{63}^{2}+A_{91} A_{63}^{4}+A_{87} A_{63}^{6}+A_{63}^{10}+A_{63}+A_{63}^{19} \\
\sigma_{40}:=A_{87}^{4} A_{63}^{4}+A_{85} A_{63}^{8}+A_{63}^{20} \\
\sigma_{42}:=A_{95} A_{63}^{4}+A_{91} A_{63}^{6}+A_{87} A_{63}^{8}+A_{63}^{12}+A_{63}^{21} \\
\sigma_{44}:=A_{87}^{4} A_{63}^{6}+A_{85} A_{63}^{10}+A_{63}^{4}+A_{63}^{22} \\
\sigma_{46}:=A_{91}^{32}+A_{95} A_{63}^{6}+A_{91} A_{63}^{8}+A_{87} A_{63}^{10}+A_{63}^{14}+A_{63}^{5}+A_{63}^{23} \\
\sigma_{48}:=A_{91}^{4}+A_{87}^{4} A_{63}^{8}+A_{85}^{2}+A_{85} A_{63}^{12}+A_{63}^{24} \\
\sigma_{50}:=A_{111}+A_{91}^{32} A_{63}^{2}+A_{95} A_{63}^{8}+A_{91} A_{63}^{10} \\
\quad+A_{87} A_{63}^{12}+A_{63}^{16}+A_{63} A_{85}^{2}+A_{63}^{25} \\
\sigma_{52}:=A_{63}^{8}+A_{87}^{2}+A_{63}^{26}+A_{91}^{4} A_{63}^{2} \\
\quad+A_{87}^{4} A_{63}^{10}+A_{85} A_{63}^{14} \\
\sigma_{54}:=1+A_{63}^{9}+A_{91}^{32} A_{63}^{4}+A_{63}^{27}+A_{95} A_{63}^{10}+A_{91} A_{63}^{12} \\
\quad+A_{87} A_{63}^{14}+A_{63} A_{87}^{2}+A_{111} A_{63}^{2}+A_{63}^{18} \\
\sigma_{56}:=A_{91}^{4} A_{63}^{4}+A_{87}^{16}+A_{63}^{28}+A_{87}^{4} A_{63}^{12}+A_{85}^{2} A_{63}^{4}+A_{85} A_{63}^{16} \\
\sigma_{58}:=A_{111} A_{63}^{4}+A_{119}+A_{63}^{29}+A_{91}^{32} A_{63}^{6}+A_{95} A_{63}^{12} \\
\quad+A_{91} A_{63}^{14}+A_{87} A_{63}^{16} A+A_{85}^{2} A_{63}^{5}+A_{63}^{20} \\
\sigma_{60}:=A_{85}+A_{91}^{2}+A_{87}^{2} A_{63}^{4}+A_{85} A_{63}^{18}+A_{87}^{16} A_{63}^{2} \\
\quad+A_{91}^{4} A_{63}^{6}+A_{87}^{4} A_{63}^{14}+A_{63}^{30}+A_{63}^{12} .
\end{aligned}
$$

The other values are $\sigma_{0}=1$, by definition, and $\sigma_{i}=0$ for odd $i$, given by the first $\delta$ identities.

After replacement of the $\sigma_{i}$ 's by their values, the remaining equations are sorted in terms of increasing size of the number of monomials:

$186,190,188,194,198,192,202,123,184,189,191,196$

$206,254,127,195,200,210,135,193,187,199,214,125$,

$131,204,252,197,222,203,238,129,139,208,250,143$,

$201,218,133,137,226,230,246,248,234,185,212,242$,

$207,141,181,216,236,244,151,183,205,220,232,147$,

$224,159,179,211,240,149,175,155,145,157,209,173$,

$163,167,228,153,171,215,253,251,165,169,161,177$,

$213,223,239,247,249,243,217,235,237,245,219,221$,

$229,231,233,227,225,241$.

We will proceed as follows.

- We successively check the equations in the order given above, up to a "solvable" one.

- After solving one equation, we restart from the beginning.

(At each stage we substitute all the known $A_{i}$ 's in the current equation, and we simplify it as much as possible.)

We give here, in order of resolution, all the "solvable" equations, and the way we used them:

$$
\begin{gathered}
I_{186}: A_{87}^{8}+A_{85}^{2} A_{63}^{8}+A_{85} A_{63}^{20}+A_{87}^{16} A_{63}^{4}+A_{91}^{4} A_{63}^{8} \\
+A_{87}^{4} A_{63}^{16}+A_{63}^{32}+A_{95}^{4}=0 \\
\Rightarrow A_{95}:=A_{87}^{2}+A_{85}^{2} A_{63}^{2}+A_{85} A_{63}^{5}+A_{87}^{4} A_{63} \\
+A_{91} A_{63}^{2}+A_{87} A_{63}^{4}+A_{63}^{8}
\end{gathered}
$$




$$
\begin{aligned}
& I_{188}: A_{87}^{8} A_{63}+A_{91}^{2} A_{63}^{3}+A_{87}^{2} A_{63}^{7}+A_{85}^{2}+A_{85}^{2} A_{63}^{9} \\
& +A_{91}+A_{87} A_{63}^{2}+A_{63}^{6}+A_{63}^{15}+A_{127}=0 \\
& \Rightarrow A_{127}:=A_{87}^{8} A_{63}+A_{91}^{2} A_{63}^{3}+A_{87}^{2} A_{63}^{7}+A_{85}^{2} \\
& +A_{85}^{2} A_{63}^{9}+A_{91}+A_{87} A_{63}^{2}+A_{63}^{6}+A_{63}^{15} \\
& I_{194}: A_{85}^{3}+1=0 \Rightarrow A_{85} \neq 0 \\
& I_{198}: A_{85} A_{87}^{2}+A_{63}^{2}=0 \Rightarrow A_{87}:=A_{63} A_{85} \\
& I_{187}: 1+A_{91} A_{85} A_{63}^{18}+A_{85}^{2} A_{91} A_{63}^{6}+A_{91}^{4} A_{63}^{21}+A_{63}^{2} \\
& +A_{63}^{142}+A_{63}^{130} A_{85}+A_{111}^{128} A_{63}^{2}+A_{91}^{16} A_{63}^{3} \\
& +A_{85}^{2} A_{63}^{39}+A_{119}^{8}+A_{91}^{6} A_{63}^{9}+A_{91}^{2} A_{63}^{33}+A_{91}^{4} A_{63}^{3} \\
& +A_{85}^{2} A_{91}^{2}+A_{85}^{2} A_{63}^{21}+A_{63}^{36}+A_{63}^{15} A_{85}+A_{91} A_{63}^{30} \\
& +A_{91}^{3}+A_{85} A_{63}^{24}+A_{85}^{2} A_{63}^{15} A_{91}^{4}+A_{91}^{2} A_{63}^{21} A_{85} \\
& +A_{63}^{9} A_{85} A_{91}^{4}+A_{91}^{4} A_{63}^{12}+A_{91}^{2} A_{63}^{6}+A_{91}^{5} A_{63}^{6} \\
& +A_{85}^{2} A_{91}^{4} A_{63}^{6}+A_{85}^{2} A_{63}^{30}+A_{63}^{45} \\
& +A_{91} A_{85}+A_{91} A_{63}^{12}+A_{63}^{3} A_{85}^{2}=0 \\
& \Rightarrow A_{119}:=1+A_{111}^{16} A_{63}^{64}+A_{85} A_{63}^{228}+A_{91}^{160} A_{63}^{192} \\
& +A_{91}^{32} A_{85}^{2} A_{63}^{66}+A_{63}^{64}+A_{85} A_{63}^{225} A_{91}^{128} \\
& +A_{63}^{80} A_{85}^{2}+A_{63}^{225} A_{85}^{2}+A_{91}^{32} A_{63}^{195}+A_{85} A_{63}^{195} \\
& +A_{91}^{2} A_{63}^{96}+A_{91}^{64} A_{85}+A_{63}^{132}+A_{91}^{128} A_{63}^{96} \\
& +A_{91}^{192} A_{63}^{33}+A_{91}^{64} A_{63}^{162} A_{85}^{2}+A_{63}^{96} A_{85} \\
& +A_{63}^{33} A_{85}^{2} A_{91}^{128}+A_{85} A_{91}^{128} A_{63}^{192}+A_{91}^{32} A_{63}^{129} \\
& +A_{63}^{209}+A_{91}^{128} A_{63}^{162}+A_{63}^{165}+A_{85} A_{91}^{32} A_{63}^{192} \\
& +A_{63}^{3} A_{85}^{2}+A_{91}^{128} A_{63}^{129}+A_{91}^{64} A_{63}^{36}+A_{85} A_{63}^{162} \\
& +A_{91}^{64} A_{63}^{192}+A_{91}^{96}+A_{91}^{32} A_{85}^{2} \\
& I_{189}: A_{91}^{2} A_{63}^{34}+A_{91}^{4} A_{85} A_{63}^{10}+A_{91} A_{63}^{31}+A_{63} A_{91}^{3} \\
& +A_{91}^{4} A_{63}^{4}+A_{85} A_{63}^{25}+A_{85}^{2} A_{63}^{22}+A_{91} A_{85} A_{63}^{19} \\
& +A_{63}^{7} A_{91}^{2}+A_{91}^{4} A_{63}^{13}+A_{91}^{5} A_{63}^{7}+A_{85}^{2} A_{63}^{31} A_{63} \\
& +A_{63}^{2}+A_{63}^{128} A_{91}+A_{63}^{37}+A_{111}^{4}+A_{91} A_{63}^{13} \\
& +A_{91}^{4} A_{63}^{22}+A_{63}^{128} A_{85}^{2}+A_{91}^{2} A_{63} A_{85}^{2}+A_{85}^{2} A_{63}^{4} \\
& +A_{85} A_{63}^{16}+A_{63}^{3}+A_{63}^{134}+A_{85}^{2} A_{63}^{40}+A_{91}^{6} A_{63}^{10} \\
& +A_{63}^{46}+A_{85}^{2} A_{91} A_{63}^{7}+A_{85}^{2} A_{63}^{16} A_{91}^{4}+A_{91}^{2} A_{63}^{22} A_{85} \\
& +A_{85}^{2} A_{91}^{4} A_{63}^{7}+A_{63} A_{91} A_{85}=0 \\
& \Rightarrow A_{111}:=A_{91} A_{85}^{2} A_{63}^{4}+A_{63}^{133} \dot{A}_{85}^{2}+A_{91} A_{85} A_{63}^{130} \\
& +A_{91}^{64} A_{85} A_{63}^{196}+A_{91}^{128} A_{63}^{64} A_{85}^{2}+A_{85}^{2} A_{91}^{64} A_{63}^{193} \\
& +A_{63}^{70} A_{85}+A_{63}^{64}+A_{63}^{128}+A_{63}^{139}+A_{85}^{2} A_{63}^{10} \\
& +A_{91}^{129} A_{63}^{130}+A_{91}^{128} A_{63}^{133} A_{85}+A_{85}^{2} A_{91} A_{63}^{193} \\
& +A_{63} A_{85}^{2}+A_{63}^{32} A_{85}^{2}+A_{63}^{64} A_{91}^{64} A_{85}+A_{63}^{73} \\
& +A_{91} A_{63}+A_{85} A_{63}^{4}+A_{63}^{133} A_{91}+A_{91}^{65} A_{63}^{193} \\
& +A_{85}^{2} A_{63}^{199}+A_{91}^{64} A_{63}^{67}+A_{63}^{161}+A_{91}^{64} A_{63}^{199} \\
& +A_{91} A_{63}^{67}+A_{91}^{128} A_{63}^{136}+A_{63}^{193} A_{91}^{128}+A_{63}^{32} A_{91}^{64} \\
& +A_{63}^{192}+A_{63}^{64} A_{91}^{192} \\
& \text { - } I_{199}: A_{91}^{16}=0 \Rightarrow A_{91}:=0 \\
& I_{203}: 1=0 \text {. } \\
& I_{61}: A_{61}+\sigma_{2}=0 \\
& I_{63}: A_{63}+A_{61} \sigma_{2}+\sigma_{4}=0 \\
& I_{65}: A_{63} \sigma_{2}+A_{61} \sigma_{4}+\sigma_{6}=0 \\
& I_{67}: A_{63} \sigma_{4}+A_{61} \sigma_{6}+\sigma_{8}=0 \\
& I_{69}: A_{63} \sigma_{6}+A_{61} \sigma_{8}+\sigma_{10}=0 \\
& I_{71}: A_{63} \sigma_{8}+A_{61} \sigma_{10}+\sigma_{12}=0 \\
& I_{73}: A_{63} \sigma_{10}+A_{61} \sigma_{12}+\sigma_{14}=0 \\
& I_{75}: A_{63} \sigma_{12}+A_{61} \sigma_{14}+\sigma_{16}=0 \\
& I_{77}: A_{63} \sigma_{14}+A_{61} \sigma_{16}+\sigma_{18}=0 \\
& I_{79}: A_{61}^{64}+A_{63} \sigma_{16}+A_{61} \sigma_{18}+\sigma_{20}=0 \\
& I_{81}: A_{61}^{64} \sigma_{2}+A_{63} \sigma_{18}+A_{61} \sigma_{20}+\sigma_{22}=0 \\
& I_{83}: A_{61}^{64} \sigma_{4}+A_{63} \sigma_{20}=A_{61} \sigma_{22}+\sigma_{24}=0 \\
& I_{85}: A_{85}+A_{61}^{64} \sigma_{6}+A_{63} \sigma_{22}+A_{61} \sigma_{24}+\sigma_{26}=0 \\
& I_{87}: A_{87}+A_{85} \sigma_{2}+A_{61}^{64} \sigma_{8}+A_{63} \sigma_{24}+A_{61} \sigma_{26}+\sigma_{28}=0 \\
& I_{89}: A_{87} \sigma_{2}+A_{85} \sigma_{4}+A_{61}^{64} \sigma_{10}+A_{63} \sigma_{26}+A_{61} \sigma_{28}+\sigma_{30}=0 \\
& I_{91}: A_{91}+A_{87} \sigma_{4}+A_{85} \sigma_{6}+A_{61}^{64} \sigma_{12}+A_{63} \sigma_{28} \\
& +A_{61} \sigma_{30}+\sigma_{32}=0 \\
& I_{93}: A_{87}^{4}+A_{91} \sigma_{2}+A_{87} \sigma_{6}+A_{85} \sigma_{8}+A_{61}^{64} \sigma_{14}+A_{63} \sigma_{30} \\
& +A_{61} \sigma_{32}+\sigma_{34}=0 \\
& I_{95}: A_{95}+A_{87}^{4} \sigma_{2}+A_{91} \sigma_{4}+A_{87} \sigma_{8}+A_{85} \sigma_{10}+A_{61}^{64} \sigma_{16} \\
& +A_{63} \sigma_{32}+A_{61} \sigma_{34}+\sigma_{36}=0 \\
& I_{97}: A_{95} \sigma_{2}+A_{87}^{4} \sigma_{4}+A_{91} \sigma_{6}+A_{87} \sigma_{10}+A_{85} \sigma_{12}+A_{61}^{64} \sigma_{18} \\
& +A_{63} \sigma_{34}+A_{61} \sigma_{36}+\sigma_{38}=0 \\
& I_{99}: A_{95} \sigma_{4}+A_{87}^{4} \sigma_{6}+A_{91} \sigma_{8}+A_{87} \sigma_{12}+A_{85} \sigma_{14}+A_{61}^{64} \sigma_{20} \\
& +A_{63} \sigma_{36}+A_{61} \sigma_{38}+\sigma_{40}=0 \\
& I_{101}: A_{95} \sigma_{6}+A_{87}^{4} \sigma_{8}+A_{91} \sigma_{10}+A_{87} \sigma_{14}+A_{85} \sigma_{16}+A_{61}^{64} \sigma_{22} \\
& +A_{63} \sigma_{38}+A_{61} \sigma_{40}+\sigma_{42}=0 \\
& I_{103}: 1+A_{95} \sigma_{8}+A_{87}^{4} \sigma_{10}+A_{91} \sigma_{12}+A_{87} \sigma_{16}+A_{85} \sigma_{18} \\
& +A_{61}^{64} \sigma_{24}+A_{63} \sigma_{40}+A_{61} \sigma_{42}+\sigma_{44}=0
\end{aligned}
$$




$$
\begin{aligned}
& I_{105}: \sigma_{2}+A_{95} \sigma_{10}+A_{87}^{4} \sigma_{12}+A_{91} \sigma_{14}+A_{87} \sigma_{18}+A_{85} \sigma_{20} \\
& +A_{61}^{64} \sigma_{26}+A_{63} \sigma_{42}+A_{61} \sigma_{44}+\sigma_{46}=0 \\
& I_{107}: A_{91}^{32}+\sigma_{4}+A_{95} \sigma_{12}+A_{87}^{4} \sigma_{14}+A_{91} \sigma_{16}+A_{87} \sigma_{20} \\
& +A_{85} \sigma_{22}+A_{61}^{64} \sigma_{28}+A_{63} \sigma_{44}+A_{61} \sigma_{46}+\sigma_{48}=0 \\
& I_{109}: A_{91}^{4}+A_{91}^{32} \sigma_{2}+\sigma_{6}+A_{95} \sigma_{14}+A_{87}^{4} \sigma_{16}+A_{91} \sigma_{18} \\
& +A_{87} \sigma_{22}+A_{85} \sigma_{24}+A_{61}^{64} \sigma_{30}+A_{63} \sigma_{46} \\
& +A_{61} \sigma_{48}+\sigma_{50}=0 \\
& +A_{63} \sigma_{48}+A_{61} \sigma_{50}+\sigma_{52}=0 \\
& +A_{61}^{64} \sigma_{34}+A_{63} \sigma_{50}+A_{61} \sigma_{52}+\sigma_{54}=0 \\
& +A_{61}^{64} \sigma_{36}+A_{63} \sigma_{52}+A_{61} \sigma_{54}+\sigma_{56}=0 \\
& +A_{85} \sigma_{32}+A_{61}^{64} \sigma_{38}+A_{63} \sigma_{54}+A_{61} \sigma_{56}+\sigma_{58}=0
\end{aligned}
$$

which gives us the following values for the $\sigma_{i}$ 's:

$$
\begin{aligned}
\sigma_{2}:= & A_{61} \\
\sigma_{4}:= & A_{61}^{2}+A_{63} \\
\sigma_{6}:= & A_{61}^{3} \\
\sigma_{8}:= & A_{63} A_{61}^{2}+A_{63}^{2}+A_{61}^{4} \\
\sigma_{10}:= & A_{61} A_{63}^{2}+A_{61}^{5} \\
\sigma_{12}:= & A_{63}^{3}+A_{63} A_{61}^{4}+A_{61}^{6} \\
\sigma_{14}:= & A_{61}^{7} \\
\sigma_{16}:= & A_{63}^{4}+A_{63}^{2} A_{61}^{4}+A_{63} A_{61}^{6}+A_{61}^{8} \\
\sigma_{18}:= & A_{61} A_{63}^{4}+A_{63}^{2} A_{61}^{5}+A_{61}^{9} \\
\sigma_{20}:= & A_{61}^{64}+A_{63}^{5}+A_{63}^{3} A_{61}^{4}+A_{63} A_{61}^{8}+A_{61}^{2} A_{63}^{4}+A_{61}^{10} \\
\sigma_{22}:= & A_{61}^{3} A_{63}^{4}+A_{61}^{11} \\
\sigma_{24}:= & A_{61}^{66}+A_{63}^{6}+A_{63}^{2} A_{61}^{8}+A_{61}^{2} A_{63}^{5}+A_{63} A_{61}^{10}+A_{61}^{12} \\
\sigma_{26}:= & A_{85}+A_{61} A_{63}^{6}+A_{63}^{2} A_{61}^{9}+A_{61}^{13} \\
\sigma_{28}:= & A_{87}+A_{61}^{64} A_{63}^{2}+A_{61}^{68}+A_{63}^{7}+A_{63}^{3} A_{61}^{8}+A_{63} A_{61}^{12}+A_{61}^{14} \\
\sigma_{30}:= & A_{85} A_{61}^{2}+A_{61}^{15} \\
\sigma_{32}:= & A_{91}+A_{87} A_{61}^{2}+A_{61}^{70}+A_{63}^{8}+A_{63}^{4} A_{61}^{8}+A_{63}^{2} A_{61}^{12} \\
& +A_{63} A_{61}^{14}+A_{61}^{16} \\
\sigma_{34}:= & A_{87}^{4}+A_{85} A_{63}^{2}+A_{85} A_{61}^{4}+A_{61}^{13}+A_{61}^{17} \\
& +A_{63}^{4} A_{61}^{9} \\
\hline &
\end{aligned}
$$

$$
\begin{aligned}
& \sigma_{36}:=A_{95}+A_{61}^{18}+A_{61}^{72}+A_{63}^{9}+A_{91} A_{61}^{2}+A_{87} A_{63}^{2}+A_{87} A_{61}^{4} \\
& +A_{61}^{64} A_{63}^{4}+A_{61}^{68} A_{63}^{2}+A_{63}^{5} A_{61}^{8}+A_{63}^{3} A_{61}^{12}+A_{63} A_{61}^{16} \\
& +A_{61}^{2} A_{63}^{8}+A_{63}^{4} A_{61}^{10} \\
& \sigma_{38}:=A_{85} A_{61}^{6}+A_{61}^{3} A_{63}^{8}+A_{63}^{4} A_{61}^{11}+A_{61}^{19}+A_{87}^{4} A_{61}^{2} \\
& \sigma_{40}:=A_{61}^{128}+A_{63}^{6} A_{61}^{8}+A_{63}^{2} A_{61}^{16}+A_{61}^{2} A_{63}^{9}+A_{63}^{5} A_{61}^{10} \\
& +A_{61}^{4} A_{63}^{8}+A_{61}^{66} A_{63}^{4}+A_{95} A_{61}^{2}+A_{91} A_{63}^{2}+A_{91} A_{61}^{4} \\
& +A_{61}^{74}+A_{63}^{10}+A_{61}^{20}+A_{87} A_{61}^{6}+A_{63} A_{61}^{18} \\
& \sigma_{42}:=A_{85} A_{63}^{2} A_{61}^{4}+A_{87}^{4} A_{63}^{2}+A_{87}^{4} A_{61}^{4}+A_{85} A_{63}^{4}+A_{85} A_{61}^{8} \\
& +A_{63}^{6} A_{61}^{9}+A_{63}^{2} A_{61}^{17}+A_{61}^{5} A_{63}^{8}+A_{61} A_{63}^{10}+A_{61}^{129}+A_{61}^{21} \\
& \sigma_{44}:=1+A_{87} A_{63}^{2} A_{61}^{4}+A_{63} A_{61}^{128}+A_{61}^{22}+A_{61}^{76}+A_{63}^{11} \\
& +A_{95} A_{63}^{2}+A_{95} A_{61}^{4}+A_{91} A_{61}^{6}+A_{87} A_{63}^{4}+A_{87} A_{61}^{8} \\
& +A_{61}^{64} A_{63}^{6}+A_{61}^{72} A_{63}^{2}+A_{63}^{7} A_{61}^{8}+A_{63}^{3} A_{61}^{16}+A_{61}^{4} A_{63}^{9} \\
& +A_{63} A_{61}^{20}+A_{61}^{6} A_{63}^{8} \\
& \sigma_{46}:=A_{85} A_{61}^{2} A_{63}^{4}+A_{61}^{7} A_{63}^{8}+A_{61}^{23}+A_{87}^{4} A_{61}^{6}+A_{85} A_{61}^{10} \\
& \sigma_{48}:=A_{91} A_{63}^{2} A_{61}^{4}+A_{87} A_{61}^{2} A_{63}^{4}+A_{63}^{12}+A_{61}^{24}+A_{61}^{132}+A_{61}^{78} \\
& +A_{61}^{2}+A_{91}^{32}+A_{95} A_{61}^{6}+A_{91} A_{63}^{4}+A_{91} A_{61}^{8}+A_{87} A_{61}^{10} \\
& +A_{63} A_{61}^{22}+A_{63}^{4} A_{61}^{16}+A_{61}^{4} A_{63}^{10}+A_{63}^{2} A_{61}^{20}+A_{61}^{6} A_{63}^{9} \\
& \sigma_{50}:=A_{91}^{4}+A_{85} A_{61}^{12}+A_{61} A_{63}^{12}+A_{63}^{4} A_{61}^{17}+A_{61}^{5} A_{63}^{10} \\
& +A_{63}^{2} A_{61}^{21}+A_{85} A_{63}^{2} A_{61}^{8}+A_{87}^{4} A_{63}^{2} A_{61}^{4}+A_{61}^{25}+A_{61}^{133} \\
& +A_{87}^{4} A_{63}^{4}+A_{87}^{4} A_{61}^{8}+A_{85} A_{63}^{6} \\
& \sigma_{52}:=A_{61}^{4}+A_{63}^{2}+A_{61}^{64} A_{63}^{8}+A_{61}^{72} A_{63}^{4}+A_{61}^{76} A_{63}^{2}+A_{63} A_{61}^{24} \\
& +A_{63} A_{61}^{132}+A_{63}^{5} A_{61}^{16}+A_{61}^{4} A_{63}^{11}+A_{63}^{3} A_{61}^{20}+A_{61}^{2} A_{63}^{12} \\
& +A_{63}^{4} A_{61}^{18}+A_{61}^{80}+A_{63}^{13}+A_{61}^{26}+A_{95} A_{63}^{2} A_{61}^{4} \\
& +A_{91} A_{61}^{2} A_{63}^{4}+A_{87} A_{63}^{2} A_{61}^{8}+A_{95} A_{63}^{4}+A_{87} A_{61}^{12} \\
& +A_{91}^{32} A_{61}^{2}+A_{95} A_{61}^{8}+A_{87} A_{63}^{6}+A_{91} A_{61}^{10}+A_{85}^{2}+A_{111} \\
& \sigma_{54}:=A_{87}^{4} A_{61}^{2} A_{63}^{4}+A_{85} A_{61}^{14}+A_{61} A_{85}^{2}+A_{61}^{3} A_{63}^{12}+A_{63}^{4} A_{61}^{19} \\
& +A_{61}^{27}+A_{87}^{4} A_{61}^{10}+A_{91}^{4} A_{61}^{2} \\
& \sigma_{56}:=A_{91} A_{63}^{2} A_{61}^{8}+A_{95} A_{61}^{2} A_{63}^{4}+A_{63}^{14}+A_{87}^{2}+A_{61}^{82}+A_{61}^{136} \\
& +A_{61}^{28}+A_{111} A_{61}^{2}+A_{91}^{32} A_{63}^{2}+A_{91}^{32} A_{61}^{4}+A_{95} A_{61}^{10} \\
& +A_{91} A_{63}^{6}+A_{91} A_{61}^{12}+A_{87} A_{61}^{14}+A_{61}^{128} A_{63}^{4}+A_{61}^{66} A_{63}^{8} \\
& +A_{61}^{74} A_{63}^{4}+A_{63} A_{85}^{2}+A_{63}^{2} A_{61}^{24}+A_{63}^{6} A_{61}^{16}+A_{61}^{2} A_{63}^{13} \\
& +A_{63}^{5} A_{61}^{18}+A_{63} A_{61}^{26}+A_{61}^{6} \\
& \sigma_{58}:=A_{85} A_{63}^{2} A_{61}^{12}+A_{85} A_{63}^{4} A_{61}^{8}+A_{87}^{4} A_{63}^{2} A_{61}^{8}+A_{87}^{16} \\
& +A_{61}^{129} A_{63}^{4}+A_{61} A_{63}^{14}+A_{61} A_{87}^{2}+A_{63}^{2} A_{61}^{25}+A_{63}^{6} A_{61}^{17} \\
& +A_{61}^{137}+A_{61}^{29}+A_{91}^{4} A_{63}^{2}+A_{91}^{4} A_{61}^{4}+A_{87}^{4} A_{63}^{6}+A_{87}^{4} A_{61}^{12} \\
& +A_{85} A_{63}^{8}+A_{85} A_{61}^{16} \text {. }
\end{aligned}
$$


The other values are $\sigma_{0}=1$, by definition, and $\sigma_{i}=0$ for odd $i$, given by the first identities.

After replacement of the $\sigma_{i}$ 's by their values, the remaining equations will be sorted in increasing size (number of monomials) order:

$180,188,196,192,186,184,204,200,190,252,189,119$, $178,182,187,212,121,194,208,220,236,198,125,185$, $244,191,193,202,248,197,216,228,224,123,133,195$, $129,206,232,250,240,205,137,141,201,181,183,246$, $254,218,127,199,210,173,179,203,157,253,131,214$, $177,149,171,234,249,139,145,153,222,242,230,135$, $251,169,238,245,155,165,213,221,209,207,237,241$, $217,226,147,175,161,143,243,163,151,211,167,247$, $233,235,219,159,229,225,239,227,215,231,223$.

We will proceed as follows.

- We successively check the equations in the order given above, up to a "solvable" one;

- After solving one equation, we restart from the beginning.

(at each stage we substitute all the known $A_{i}$ 's, and we show the most simple equation possible.)

We will first show that $A_{61} \neq 0$.

Suppose that $A_{61}=0$. Then

$$
\begin{aligned}
I_{196}: A_{85}^{3}=0 & \Rightarrow A_{85}:=0, \\
I_{208}: A_{87}^{6}=0 & \Rightarrow A_{87}:=0, \\
I_{236}: 1 & =0,
\end{aligned}
$$

so $A_{61} \neq 0$.

We give here, in order of resolution, all the "solvable" equations, and the way we used them:

$$
\begin{gathered}
I_{180}: A_{61}^{8} A_{85} A_{63}^{4}+A_{85} A_{63}^{8}+A_{61}^{4} A_{91}^{4}+A_{61}^{29} \\
+A_{61}^{3} A_{85}^{2}+A_{61}^{16} A_{85}+A_{61}^{12} A_{87}^{4}+A_{87}^{16}=0 \\
\Rightarrow_{91}:=A_{61} A_{85} A_{63}+A_{61}^{254} A_{85} A_{63}^{2}+A_{61}^{2} A_{87}+A_{61}^{70} \\
+A_{61}^{191} A_{85}^{2}+A_{61}^{3} A_{85}+A_{61}^{254} A_{87}^{4} \\
I_{196}: A_{95}^{2} A_{61}^{3}+A_{87}^{8} A_{61} A_{63}^{2}+A_{85}^{2} A_{61} A_{63}^{6}+A_{87}^{2} A_{61}^{3} A_{63}^{4} \\
+A_{85}^{3}+A_{61}^{131}+A_{63}^{8}+A_{61}^{139} A_{63}^{4} \\
+A_{61}^{130} A_{87}^{4}+A_{61}^{5} A_{85}^{2} A_{63}^{4}=0 \\
A_{95}:=A_{61}^{126} A_{85}^{3}+A_{61}^{254} A_{87}^{4} A_{63}+A_{61}^{254} A_{85} A_{63}^{3}+A_{87} A_{63}^{2} \\
+A_{61} A_{85} A_{63}^{2}+A_{61}^{64} A_{63}^{4}+A_{61}^{68} A_{63}^{2}+A_{61}^{191} A_{87}^{2} \\
I_{192}: A_{85}^{3}=0 \Rightarrow A_{85}=0 \\
I_{200}: 1=0 .
\end{gathered}
$$

APPENDIX C

$B(511,123)$ has Minimum Distance $>123$

We consider Newton's identities for $0<i \leq n=511$ for the code $B(511,123)$, and for the weight $\delta=123$. We want to prove that there exists no codeword of weight $\delta$.

The nonzero power sum symmetric functions of the code are

$$
A_{123}, A_{125}, A_{127}, A_{171}, A_{175}, A_{183}, A_{187} \text {, }
$$

$A_{191}, A_{219}, A_{223}, A_{239}, A_{255}$.
Since 511 and 123 are relatively prime we can suppose $A_{123}=1$. We will give an abbreviated proof for this code (the complete proof is too long to present here).

We will first solve the linear triangular system giving the $\sigma_{i}$ 's for even $i$ as polynomials depending on the nonzero $A_{i}$ 's. The $\sigma_{i}$ 's for odd $i$ are null. We consider that the $\sigma_{i}$ 's have been substituted in the equations.

Furthermore we will suppose $A_{125} \neq 0$ (when $A_{125}=0$, we found a contradiction).

We give here the equations we used for the resolution, and the way we used them:

$$
\begin{aligned}
I_{372}: & A_{125}^{63}+A_{171}^{8} A_{125}^{13}+A_{171}^{32} A_{125}^{5}+A_{171}^{4} A_{127}^{8} A_{125}^{22} \\
& +A_{171}^{4} A_{125}^{6} A_{127}^{16}+A_{187}^{4} A_{125}^{6}+A_{175}^{16} A_{125}^{2}+A_{183}^{4} A_{125}^{14} \\
& +A_{171}^{4} A_{125}^{38}+A_{171}^{16} A_{127}^{8} A_{125}^{18}+A_{171}^{16} A_{125}^{2} A_{127}^{16} \\
& +A_{171}^{16} A_{127}^{4} A_{125}^{26}+A_{171}^{16} A_{125}^{34}+A_{175}^{4} A_{125}^{30} \\
& +A_{171}^{64} A_{125}^{2} A_{127}^{8}+A_{171}^{64} A_{127}^{4} A_{125}^{10}+A_{171}^{64} A_{125}^{18}=0
\end{aligned}
$$

$$
\begin{aligned}
=A_{187}:= & A_{125}^{142}+A_{125}^{385} A_{171}^{2}+A_{125}^{383} A_{171}^{8}+A_{171} A_{127}^{2} A_{125}^{4} \\
& +A_{171} A_{127}^{4}+A_{125}^{3} A_{171}^{4} A_{127}^{2}+A_{125}^{510} A_{175}^{4} \\
& +A_{183} A_{125}^{2}+A_{171} A_{125}^{8} \\
& +A_{175} A_{125}^{6}+A_{125}^{510} A_{171}^{4} A_{127}^{4} \\
& +A_{125}^{5} A_{171}^{4} A_{127}+A_{171}^{4} A_{125}^{7}+A_{125}^{510} A_{171}^{16} A_{127}^{2} \\
& +A_{125} A_{171}^{16} A_{127}+A_{171}^{16} A_{125}^{3}
\end{aligned}
$$

$$
\begin{aligned}
& I_{388}: A_{171}^{8} A_{127}^{10}+A_{125}^{258} A_{127}^{16}+A_{175}^{4} A_{125}^{257}+A_{125}^{274} A_{127}^{8} \\
&+A_{125}^{282} A_{127}^{4}+A_{171}^{4} A_{125}^{257} A_{127}^{4}+A_{175}^{2} A_{127}^{4} A_{125}^{10} \\
&+A_{171}^{8} A_{127}^{6} A_{125}^{8}+A_{171}^{32} A_{127}^{6}+A_{175}^{2} A_{125}^{2} A_{127}^{8} \\
&+ A_{175}^{8} A_{127}^{2}+A_{183}^{2} A_{125}^{2} A_{127}^{4}+A_{191}^{2} A_{125}^{2} \\
&+ A_{125}^{4} A_{171}^{32} A_{127}^{4}+A_{125}^{12} A_{171}^{8} A_{127}^{4}+A_{125}^{14} A_{171}^{2} A_{127}^{4}=0 \\
&=A_{191}:=A_{125}^{510} A_{171}^{4} A_{127}^{5}+A_{125}^{128} A_{127}^{8}+A_{125}^{383} A_{175}^{2}+A_{125}^{136} A_{127}^{4} \\
&+A_{125}^{140} A_{127}^{2}+A_{125}^{383} A_{171}^{2} A_{127}^{2}+A_{175} A_{127}^{2} A_{125}^{4} \\
&+A_{125}^{3} A_{171}^{4} A_{127}^{3}+A_{125}^{510} A_{171}^{16} A_{127}^{3}+A_{175} A_{127}^{4} \\
&+A_{125}^{510} A_{175}^{4} A_{127}+A_{183}^{4} A_{127}^{2}+A_{171}^{16} A_{125} A_{127}^{2} \\
&+A_{171}^{4} A_{127}^{2} A_{125}^{5}+A_{125}^{6} A_{171} A_{127}^{2} \\
& I_{392}: A_{125}^{2}+A_{171}^{6}=0 \Rightarrow A_{171}:=A_{125}^{341} \\
& I_{404}: A_{127}^{2}+A_{125}^{340} A_{175}^{2}=0 \Rightarrow A_{175}:=A_{127}^{341} A_{125}^{341} \\
& I_{412}:=A_{125}^{97}+1=0 \Rightarrow A_{125}:=1
\end{aligned}
$$$$
I_{420}: A_{127}^{2}+A_{183}^{2}+A_{183}^{4}=0 \Rightarrow A_{127}:=A_{183}+A_{183}^{2}
$$$$
I_{428}: 1=0 \text {. }
$$ 


\section{ACKNOWLEDGMENT}

The authors wish to thank E. F. Assmus, G. D. Cohen, and H. F. Mattson for enriching discussions and valuable suggestions. They would also like to thank G. Norton for his careful and patient reading which improved the paper.

\section{REFERENCES}

[1] E. F. Assmus and J. D. Key, "Affine and projective planes," Discrete Math., vol. 83, pp. 161-187, 1990.

[2] P. Charpin, "Codes cycliques étendus affines-invariants et antichaínes d'un ensemble partiellement ordonné," Discrete Math., vol. 80, pp. 229-247, 1990.

[3] G. Cohen, "Distance Minimale et Enumération des Poids des Codes Linéaires," Thèse de $3^{\mathrm{e}}$ Cycle, ENST, Apr. 1976.

[4] - " "On the minimum distance of some BCH codes," IEEE Trans. Inform. Theory, vol. 26, p. 363, May 1980.

[5] J.-L. Dornstetter, "Quelques resultats sur les codes BCH binaires en longueur 255," ENST stage rep., Annex, July 1982.
[6] H. J. Helgert and R. D. Stinaff, "Shortened BCH codes," IEEE Trans. Inform. Theory, vol. 19, pp. 818-820, Nov. 1973.

[7] T. Kasami and S. Lin, "Some results on the minimum weight of primitive BCH codes," IEEE Trans. Inform. Theory, vol. 18, pp. 824-825, Nov. 1972.

[8] T. Kasami, S. Lin, and W. W. Peterson, "Some results on cyclic codes which are invariant under the affine group and their applications," Inform. Contr., vol. 11, pp. 475-496, 1967.

[9] -, "New generalisations of the Reed-Muller codes-Part I: Primitive codes," IEEE Trans. Inform. Theory, vol. 14, pp. 189-199, Mar. 1968.

[10] T. Kasami and N. Tokura, "Some remarks on BCH bounds and minimum weights of binary primitive BCH codes," IEEE Trans. Inform. Theory, vol. 15, pp. 408-413, May 1969.

[11] F. J. MacWilliams and N. J. A. Sloane, The Theory of Error Correcting Codes. Amsterdam: North-Holland, 1986.

[12] W. W. Peterson, Error-Correcting Codes. Cambridge, MA: MIT Press, 1961.

[13] J. H. van Lint and R. M. Wilson, "On the minimum distance of cyclic codes," IEEE Trans. Inform. Theory, vol. 32, pp. 23-40, Jan. 1986. 